\title{
Efficient Midfrequency Analysis of Built-Up Structure Systems with Interval Parameters
}

\author{
Hui Yin, Dejie Yu, Shengwen Yin, and Baizhan Xia \\ State Key Laboratory of Advanced Design and Manufacturing for Vehicle Body, Hunan University, Changsha, Hunan 410082, China
}

Correspondence should be addressed to Dejie Yu; djyu@hnu.edu.cn

Received 22 May 2015; Accepted 18 August 2015

Academic Editor: Ranjan Banerjee

Copyright (C) 2015 Hui Yin et al. This is an open access article distributed under the Creative Commons Attribution License, which permits unrestricted use, distribution, and reproduction in any medium, provided the original work is properly cited.

To improve the efficiency of midfrequency analysis of built-up structure systems with interval parameters, the second-order interval and subinterval perturbation methods are introduced into the hybrid finite element/statistical energy analysis (FE/SEA) framework in this paper. Based on the FE/SEA for built-up structure systems and the second-order interval perturbation method, the response variables are expanded with the second-order Taylor series and nondiagonal elements of the Hessian matrices are neglected. Extreme values of the expanded variables are searched by using efficient search algorithm. For large parameter intervals, the subinterval perturbation method is introduced. Numerical results verify the effectiveness of the proposed methods.

\section{Introduction}

In the last two decades, predicting the response of a system with uncertainties has got more and more attention in the engineering design. There are several ways to describe the parametric uncertainties of a system, such as random variables and intervals [1-3]. If the objective information about the uncertain parameters is adequate to establish the probability density functions of them, the random variable model can be the prior way to describe the uncertainties. Many approaches have been proposed to deal with the probabilistic uncertainty recently, such as the Monte Carlo method, the spectral stochastic method, and the perturbation stochastic method $[4,5]$. Unfortunately, in the early stage of design, there may be no sufficient statistical information to establish the probability density functions of the uncertain parameters. Under this circumstance, the nonprobabilistic model, such as the interval model, may be an advisable model to represent the uncertain parameters. In this paper, the interval model is selected to describe the parametric uncertainty.

Before performing the uncertain analysis, we must select an approach to model the system. The finite element method (FEM) [6] is the most commonly used technique to model a system in engineering practice, owing to its simplicity and accuracy. However, because the computational efficiency of FEM typically decreases exponentially with the increase of frequency, it is improper to analyze mid- to high-frequency system by using FEM. Thus, the application of FEM is limited to the so-called low-frequency range [7]. Statistical energy analysis (SEA) [8] is a statistical technique that was developed specifically to solve high-frequency problems. This approach is established on the assumption that the system is highly random. In contrast with FEM, the computational efficiency of SEA model is much better due to the much fewer degrees of freedom of it.

As stated above, the low- and high-frequency problems can be efficiently solved by using FEM and SEA, respectively. But for a system consisting of both the low- and highfrequency subsystems, which is the so-called "midfrequency" system, neither method is suitable: for pure FEM, the system must be modeled by lots of degrees of freedom and the computational efficiency is too low; for pure SEA, the tenets that the system must be "highly random" may not be met. In recent years, a variety of methods have been proposed for the analysis of midfrequency system. The variational theory of complex rays (VTCR) $[9,10]$ and the wave based method (WBM) [11] are deterministic methods based on the Trefftz approach [12] for midfrequency analysis, and they are both aiming to improve the computational efficiency by 
modeling the system with fewer degrees of freedom than that of FEM. Another method for midfrequency analysis is the hybrid approach by dividing the system into deterministic subsystems and highly random subsystems, such as the fuzzy structure theory [13]. Based on this deterministic/random partitioning idea, Langley and coworkers have recently developed a hybrid finite element/statistical energy method (FE-SEA) [14]. In this proposed method, the deterministic subsystem is called the "master system" and modeled by FEM; the random subsystem is called the "subsystem" and modeled by SEA. The randomness of the "subsystem" is modeled as nonparametric uncertainty. The coupling of the two systems is achieved by using a diffuse field reciprocity relation [15]. A lot of research works about FE-SEA have been done by Langley and coworkers [16-19].

As previously mentioned, the SEA subsystems of the FESEA model are assumed to be highly random, and the randomness of them is modeled as nonparametric uncertainty, while the FE components are assumed to be fully deterministic; in other words, the uncertainties of the FE components are ignored. However, uncertainties in properties caused by manufacturing imperfections or aggressive environmental factors are unavoidable, and it is important to take the uncertainties of the FE components into consideration in engineering design. Recently, Cicirello and Langley have introduced parametric uncertainty into the FE components by considering the parameters of them as probabilistic or interval rather than deterministic [20]. Thus, a hybrid uncertain model with parametric and nonparametric uncertainties is yielded. The distribution of the response of this hybrid model can be obtained by dealing with the parametric uncertainty with Monte Carlo simulations and the nonparametric uncertainty analytically. This method will be efficient when the FE components have few uncertain parameters and degrees of freedom. However, for large scale engineering systems with many uncertain parameters, it is computationally intensive to employ the Monte Carlo simulations to deal with the parametric uncertainty. Developing efficient MCS techniques [21] or alternative algorithms [22,23] is a direction to improve the efficiency of the analysis method for the hybrid model with parametric and nonparametric uncertainties. Recently, Cicirello and Langley [24] have proposed two different asymptotic statistical techniques to target this problem, namely, the hybrid FE/SEA method combined with the firstorder reliability method and the hybrid FE/SEA method combined with Laplace's method, which allow the evaluation of the failure probability of a complex built-up system with probabilistic input parameters of the FE components. The two methods are much more efficient than the FE Monte Carlo simulations and the accuracy of them was good. Another powerful tool for solving the stochastic problems is the stochastic finite element method (SFEM) [2], which mainly includes the perturbation stochastic finite element method (PSFEM) [25-27] and the spectral stochastic finite element method (SSFEM) [28]. These numerical analysis methods are all probabilistic techniques for propagating the probabilistic parametric uncertainty, while for the interval analysis, many other approaches have been proposed, such as the Gaussian elimination scheme [29], the vertex method [30], and the interval perturbation method (IPM) [31]. IPM is an efficient technique for interval analysis proposed by Qiu et al. In this method, the interval matrices and the interval vectors were expanded to a first-order Taylor series. To improve the accuracy of the IPM, an interval perturbation method based on the second-order Taylor expansion (SIPM) [32,33] was recently developed. Because of the neglect of the higher order terms of Taylor series, IPM is limited to the interval analysis with narrow parameter intervals. To release this restriction, the subinterval analysis technique was introduced into the interval perturbation method [34]. The interval and subinterval perturbation methods have been widely applied to the interval analysis of vibroacoustic response due to their simplicity and efficiency [35-37].

In this paper, to improve the efficiency of the midfrequency analysis of built-up structure systems with interval parametric uncertainty, the second-order interval perturbation method and subinterval analysis technique are introduced into the hybrid FE-SEA framework. Firstly, the second-order interval perturbation method combined with FE-SEA (SIPFEM/SEA) is proposed for the response prediction of the built-up structure systems with nonparametric and small interval parametric uncertainties; secondly, the subinterval perturbation method based on the SIPFEM/SEA is introduced to predict the response of the built-up structure systems with nonparametric and large interval parametric uncertainties. The procedure of the SIPFEM/SEA method is as follows: at first, the ensemble averaged energy of the SEA components and the cross-spectrum of the response of the FE components are expanded with the second-order Taylor series; for the sake of simplicity and efficiency, the nondiagonal elements of the Hessian matrices are neglected; then, by searching the target positions of interval parameters that maximize or minimize the objective functions, the bounds of the expanded responses can be obtained. For large parameter intervals, the subinterval perturbation method based on the SIPFEM/SEA is introduced. Effectiveness of the proposed methods is verified by the numerical results of two built-up structure models. Benchmark comparisons are made with the Monte Carlo simulations of the hybrid FE/SEA models.

\section{Basic Principle of the Hybrid FE/SEA Theory for Built-Up Structure Systems with Fixed FE Properties}

This section is intended to summarize the hybrid FE/SEA equations for built-up structure systems with fixed FE properties as presented by Langley et al. The main procedure for the hybrid FE/SEA method for built-up structure systems can be summarized as follows. At first, a built-up structure system is partitioned into the long-wavelength subsystems and the short-wavelength subsystems, which are modeled by the FEM and the SEA, respectively. Secondly, the response of each SEA subsystem is described as the superposition of a series of ingoing waves and reflection waves, which are called the "direct field" and "reverberant field," respectively. Finally, a diffuse field reciprocity relation between the reverberant 
force loading and the energy responses of the SEA subsystems are established for the coupling of the FE components and SEA subsystems. The detailed equations of the hybrid FE/SEA method will be presented in the following sections.

2.1. The Dynamic Equilibrium Equation of the FE Components. The master system consists of the FE components which can be described by a set of degrees of freedom q. For a specific frequency $\omega$, the equations of motion for the FE components can be written as

$$
\mathbf{D}_{d} \mathbf{q}=\mathbf{f}+\sum_{k} \mathbf{f}_{k}
$$

where $\mathbf{D}_{d}$ is the dynamic stiffness matrix of the FE components which can be obtained by FEM, $\mathbf{f}$ is the external force vector applied directly to the FE components, and $\mathbf{f}_{k}$ is the force vector exerted on the FE components by the subsystem $k$. $\mathbf{f}_{k}$ is considered to be the sum of two parts and can be written as

$$
\mathbf{f}_{k}=\mathbf{f}_{\mathrm{rev}}^{(k)}-\mathbf{D}_{\mathrm{dir}}^{(k)} \mathbf{q}
$$

with $\mathbf{f}_{\text {rev }}^{(k)}$ the "reverberant field force vector" arising from the reflected waves and $\mathbf{D}_{\text {dir }}^{(k)}$ being the "direct field dynamic stiffness matrix" for subsystem $k$.

By combining (1) and (2), one can get

$$
\mathbf{D}_{\text {tot }} \mathbf{q}=\mathbf{f}+\sum_{k} \mathbf{f}_{\mathrm{rev}}^{(k)}
$$

where $\mathbf{D}_{\text {tot }}$ can be expressed as

$$
\mathbf{D}_{\text {tot }}=\mathbf{D}_{d}+\sum_{k} \mathbf{D}_{\mathrm{dir}}^{(k)}
$$

2.2. The Power Balance Equation of the SEA Subsystems. The SEA subsystem is described by the ensemble averaged energy $E$, which can be calculated via the power balance equation expressed as

$$
\begin{array}{r}
\omega\left(\eta_{j}+\eta_{d, j}\right) E_{j}+\sum_{k} \omega \eta_{j k} n_{j}\left(\frac{E_{j}}{n_{j}}-\frac{E_{k}}{n_{k}}\right) \\
=P_{\mathrm{in}, j}^{\mathrm{ext}}+P_{\mathrm{in}, j}, \\
j=1,2, \ldots, N_{s},
\end{array}
$$

where $n_{j}, E_{j}$, and $\eta_{j}$ are the modal density, the ensemble averaged energy, and the loss factor of the subsystem $j$, respectively, $\eta_{d, j}$ is the coupling loss factor between the subsystem $j$ and the master system, $\eta_{j k}$ is the coupling loss factor between the subsystem $j$ and the subsystem $k$, and $N_{s}$ is the number of the SEA subsystems. $P_{\mathrm{in}, j}^{\text {ext }}$ and $P_{\text {in }, j}$ are the power input to the subsystem $j$ arising from the forces applied to the master system and directly to the subsystem $j$, respectively. $P_{\text {in, } j}$ can be calculated by the conventional SEA method, and other terms in (5) can be calculated by

$$
\begin{aligned}
\omega \eta_{d, j} & =\left(\frac{2 \alpha_{j}}{\pi n_{j}}\right) \sum_{r s} \operatorname{Im}\left\{D_{d, r s}\right\}\left(\mathbf{D}_{\text {tot }}^{-1} \operatorname{Im}\left\{\mathbf{D}_{\text {dir }}^{(j)}\right\} \mathbf{D}_{\text {tot }}^{-H}\right)_{r s} \\
\omega \eta_{j k} & =\left(\frac{2 \alpha_{k}}{\pi n_{j}}\right) \sum_{r s} \operatorname{Im}\left\{D_{\text {dir }, r s}^{(j)}\right\}\left(\mathbf{D}_{\text {tot }}^{-1} \operatorname{Im}\left\{\mathbf{D}_{\text {dir }}^{(k)}\right\} \mathbf{D}_{\text {tot }}^{-H}\right)_{r s} \\
P_{\mathrm{in}, j}^{\mathrm{ext}} & =\left(\frac{\omega}{2}\right) \sum_{r s} \operatorname{Im}\left\{D_{\mathrm{dir}, r s}^{(j)}\right\}\left(\mathbf{D}_{\mathrm{tot}}^{-1} \mathbf{S}_{f f} \mathbf{D}_{\text {tot }}^{-H}\right)_{r s},
\end{aligned}
$$

where the superscript $H$ stands for the Hermitian transpose, $\mathbf{S}_{f f}$ is the cross-spectral matrix of the external loadings $\mathbf{f}$, and $\mathbf{S}_{f f}=E\left[\mathrm{ff}^{H}\right]$ with $E[\cdot]$ being the ensemble average. $\alpha_{j}$ is a factor in consideration of the local concentrations in the wavefield, and the details about it are discussed in [38]. If the subsystem is excited predominantly through the master system, the value of $\alpha_{k}$ is close to 2 ; in other cases, $\alpha_{j}$ equals 1 .

According to [15], there is a relationship between $\eta_{j k}$ and $\eta_{k j}$, which can be expressed as

$$
\eta_{j k} n_{j}=\eta_{k j} n_{k}
$$

Thus, (5) can be rewritten as the following matrix form:

$$
\mathbf{C E}=\mathbf{P}_{\text {in }}^{\text {ext }}+\mathbf{P}_{\text {in }},
$$

where $\mathbf{E}, \mathbf{P}_{\text {in }}^{\text {ext }}$, and $\mathbf{P}_{\text {in }}$ are the vectors made up of $E_{j}, P_{\text {in }, j}^{\text {ext }}$, and $P_{\text {in, } j}\left(j=1,2, \ldots, N_{s}\right)$, respectively, and $\mathbf{C}$ is the coefficient matrix, the $j k$ th element of which can be written as

$$
\begin{aligned}
C_{j k}=\left\{\begin{aligned}
\omega\left(\eta_{j}+\eta_{d, j}+\sum_{k=1, k \neq j}^{N_{s}} \eta_{j k}\right), & j=k \\
-\omega \eta_{j k}, & j \neq k
\end{aligned}\right. \\
\left(j, k=1,2, \ldots, N_{s}\right) .
\end{aligned}
$$

2.3. The Coupling between the FE and SEA Components. As previously mentioned, the coupling between the FE and SEA components is achieved by using the diffuse field reciprocity relation, which can be expressed as

$$
\mathbf{S}_{f f, \mathrm{rev}}^{(j)}=E\left[\mathbf{f}_{\mathrm{rev}}^{(j)} \mathbf{f}_{\mathrm{rev}}^{(j) H}\right]=\frac{4 E_{j}}{\pi \omega n_{j}} \operatorname{Im}\left\{\mathbf{D}_{\mathrm{dir}}^{(j)}\right\} .
$$

By combining (3) and (12), the cross-spectrum of the response of the FE components can be calculated by

$$
\begin{aligned}
\mathbf{S}_{q q} & =E\left[\mathbf{q q}^{H}\right] \\
& =\mathbf{D}_{\text {tot }}^{-1}\left[\mathbf{S}_{f f}+\sum_{j} \frac{4 \alpha_{j} E_{j}}{\pi \omega n_{j}} \operatorname{Im}\left\{\mathbf{D}_{\text {dir }}^{(j)}\right\}\right] \mathbf{D}_{\text {tot }}^{-H} .
\end{aligned}
$$

It can be seen from (13) that the response of the FE components is controlled by both the forces applied directly to the FE components and the reverberant forces arising from the SEA subsystems. 


\section{Introducing Interval Parametric} Uncertainty into the FE Components within the Hybrid FE-SEA Model for Built-Up Structure Systems

In this section, the interval parametric uncertainty is introduced into the FE components within the hybrid FE-SEA model for built-up structure systems, and the interval formulations for the responses of the built-up structure systems are discussed as follows.

Assume that the parameter vector $\mathbf{a}^{I}$ stands for the set of the interval parameters of the FE components, and it can be written as

$$
\begin{aligned}
& \mathbf{a}^{I}=\left[\mathbf{a}^{L}, \mathbf{a}^{R}\right]=\left(a_{i}^{I}\right), \\
& a_{i}^{I}=\left[a_{i}^{L}, a_{i}^{R}\right],
\end{aligned}
$$

$$
i=1,2, \ldots, N
$$

where the subscripts $L$ and $R$ stand for the lower and upper bounds of the interval parameters, respectively. $N$ is the number of the interval parameters. Because of the interval description of the input parameters, the responses of the built-up structure systems in (5) and (13) become interval variables, which can be expressed as

$$
\begin{aligned}
E_{j}^{I} & =\left[E_{j}^{L}, E_{j}^{R}\right]=E_{j}\left(\mathbf{a}^{I}\right) \\
\mathbf{S}_{q q}^{I} & =\left[\mathbf{s}_{q q}^{L}, \mathbf{S}_{q q}^{R}\right]=\mathbf{S}_{q q}\left(\mathbf{a}^{I}\right),
\end{aligned}
$$

where

$$
\begin{aligned}
E_{j}^{L} & =\min \left\{E_{j} \mid E_{j}=E_{j}(\mathbf{a}), \mathbf{a} \in \mathbf{a}^{I}\right\} \\
E_{j}^{R} & =\max \left\{E_{j} \mid E_{j}=E_{j}(\mathbf{a}), \mathbf{a} \in \mathbf{a}^{I}\right\} \\
\mathbf{S}_{q q}^{L} & =\min \left\{\mathbf{S}_{q q} \mid \mathbf{S}_{q q}=\mathbf{S}_{q q}(\mathbf{a}), \mathbf{a} \in \mathbf{a}^{I}\right\} \\
\mathbf{S}_{q q}^{R} & =\max \left\{\mathbf{S}_{q q} \mid \mathbf{S}_{q q}=\mathbf{S}_{q q}(\mathbf{a}), \mathbf{a} \in \mathbf{a}^{I}\right\} .
\end{aligned}
$$

The bounds in (15) can be obtained by using the minimization/maximization analysis shown in (16), which can be implemented by the Monte Carlo simulations of the hybrid FE-SEA model. Also, the Monte Carlo simulations will be used to verify the effectiveness of the proposed methods discussed in the following sections.

\section{SIPFEM/SEA for the Midfrequency Analysis of Built-Up Structure Systems with Interval Parameters}

In this section, the second-order interval perturbation method is introduced into the hybrid FE-SEA framework, and the SIPFEM/SEA method is proposed for the midfrequency analysis of built-up structure systems with small interval parametric uncertainty.
4.1. Basic Formulation for SIPFEM/SEA. For the sake of convenience, (14) can be rewritten as

$$
\begin{array}{r}
\mathbf{a}^{I}=\left[\mathbf{a}^{m}-\Delta \mathbf{a}, \mathbf{a}^{m}+\Delta \mathbf{a}\right]=\mathbf{a}^{m}+\Delta \mathbf{a}^{I}=\mathbf{a}^{m}+\Delta \mathbf{a} e^{I}, \\
a_{i}^{I}=\left[a_{i}^{m}-\Delta a_{i}, a_{i}^{m}+\Delta a_{i}\right]=a_{i}^{m}+\Delta a_{i}^{I}=a_{i}^{m}+\Delta a_{i} e^{I}, \\
(i=1,2, \ldots, N),
\end{array}
$$

where

$$
\begin{aligned}
\mathbf{a}^{m} & =\left(a_{i}^{m}\right)=\frac{\left(\mathbf{a}^{L}+\mathbf{a}^{R}\right)}{2}, \\
a_{i}^{m} & =\frac{\left(a_{i}^{L}+a_{i}^{R}\right)}{2}, \\
\Delta \mathbf{a} & =\left(\Delta a_{i}\right)=\frac{\left(\mathbf{a}^{R}-\mathbf{a}^{L}\right)}{2}, \\
\Delta a_{i} & =\frac{\left(a_{i}^{R}-a_{i}^{L}\right)}{2}, \\
\Delta \mathbf{a}^{I} & =[-\Delta \mathbf{a}, \Delta \mathbf{a}], \\
\Delta a_{i}^{I} & =\left[-\Delta a_{i}, \Delta a_{i}\right], \\
e^{I} & =[-1,1] .
\end{aligned}
$$

Based on the second-order Taylor expansion, the interval response variables in (15) can be expanded about the mean value vector $\mathbf{a}^{m}$ and expressed as

$$
\begin{aligned}
E_{j}^{I}= & E_{j}\left(\mathbf{a}^{I}\right) \\
= & E_{j}\left(\mathbf{a}^{m}\right)+\sum_{i=1}^{N} \frac{\partial E_{j}\left(\mathbf{a}^{m}\right)}{\partial a_{i}} \Delta a_{i}^{I} \\
& +\frac{1}{2} \sum_{i=1}^{N} \sum_{l=1}^{N} \frac{\partial^{2} E_{j}\left(\mathbf{a}^{m}\right)}{\partial a_{i} \partial a_{l}} \Delta a_{i}^{I} \Delta a_{l}^{I}, \\
\mathbf{S}_{q q}^{I}= & \mathbf{S}_{q q}\left(\mathbf{a}^{I}\right) \\
= & \mathbf{S}_{q q}\left(\mathbf{a}^{m}\right)+\sum_{i=1}^{N} \frac{\partial \mathbf{S}_{q q}\left(\mathbf{a}^{m}\right)}{\partial a_{i}} \Delta a_{i}^{I} \\
& +\frac{1}{2} \sum_{i=1}^{N} \sum_{l=1}^{N} \frac{\partial^{2} \mathbf{S}_{q q}\left(\mathbf{a}^{m}\right)}{\partial a_{i} \partial a_{l}} \Delta a_{i}^{I} \Delta a_{l}^{I}
\end{aligned}
$$

with $E_{j}\left(\mathbf{a}^{m}\right)$ and $\mathbf{S}_{q q}\left(\mathbf{a}^{m}\right)$ being the values of $E_{j}^{I}$ and $\mathbf{S}_{q q}^{I}$ at the mean value vector $\mathbf{a}^{m}$

Equation (19) can be rewritten as

$$
\begin{aligned}
E_{j}^{I}= & E_{j}\left(\mathbf{a}^{I}\right) \\
= & E_{j}\left(\mathbf{a}^{m}\right)+\left(\mathbf{E}_{j}^{\prime}\left(\mathbf{a}^{m}\right)\right)^{T} \Delta \mathbf{a}^{I} \\
& +\frac{1}{2}\left(\Delta \mathbf{a}^{I}\right)^{T} \mathbf{E}_{j}^{\prime \prime}\left(\mathbf{a}^{m}\right) \Delta \mathbf{a}^{I},
\end{aligned}
$$


where $\mathbf{E}_{j}^{\prime}\left(\mathbf{a}^{m}\right)$ and $\mathbf{E}_{j}^{\prime \prime}\left(\mathbf{a}^{m}\right)$ are the gradient vector and the Hessian matrix at the mean value vector $\mathbf{a}^{m}$, respectively, and they can be expressed as

$$
\begin{aligned}
& \mathbf{E}_{j}^{\prime}\left(\mathbf{a}^{m}\right)=\left[\frac{\partial E_{j}^{\prime}\left(\mathbf{a}^{m}\right)}{\partial a_{1}}, \frac{\partial E_{j}^{\prime}\left(\mathbf{a}^{m}\right)}{\partial a_{2}}, \ldots, \frac{\partial E_{j}^{\prime}\left(\mathbf{a}^{m}\right)}{\partial a_{N}}\right] \\
& \mathbf{E}_{j}^{\prime \prime}\left(\mathbf{a}^{m}\right) \\
& =\left[\begin{array}{cccc}
\frac{\partial^{2} E_{j}^{\prime \prime}\left(\mathbf{a}^{m}\right)}{\partial a_{1} \partial a_{1}} & \frac{\partial^{2} E_{j}^{\prime \prime}\left(\mathbf{a}^{m}\right)}{\partial a_{1} \partial a_{2}} & \cdots & \frac{\partial^{2} E_{j}^{\prime \prime}\left(\mathbf{a}^{m}\right)}{\partial a_{1} \partial a_{N}} \\
\frac{\partial^{2} E_{j}^{\prime \prime}\left(\mathbf{a}^{m}\right)}{\partial a_{2} \partial a_{1}} & \frac{\partial^{2} E_{j}^{\prime \prime}\left(\mathbf{a}^{m}\right)}{\partial a_{2} \partial a_{2}} & \cdots & \frac{\partial^{2} E_{j}^{\prime \prime}\left(\mathbf{a}^{m}\right)}{\partial a_{2} \partial a_{N}} \\
\cdots & \cdots & \cdots & \cdots \\
\frac{\partial^{2} E_{j}^{\prime \prime}\left(\mathbf{a}^{m}\right)}{\partial a_{N} \partial a_{1}} & \frac{\partial^{2} E_{j}^{\prime \prime}\left(\mathbf{a}^{m}\right)}{\partial a_{N} \partial a_{2}} & \cdots & \frac{\partial^{2} E_{j}^{\prime \prime}\left(\mathbf{a}^{m}\right)}{\partial a_{n} \partial a_{N}}
\end{array}\right] .
\end{aligned}
$$

Similarly, the rsth element of $\mathbf{S}_{q q}^{I}$ can be written as

$$
\begin{aligned}
S_{q q, r s}^{I}= & S_{q q, r s}\left(\mathbf{a}^{I}\right) \\
= & S_{q q, r s}\left(\mathbf{a}^{m}\right)+\left(\mathbf{s}_{q q, r s}^{\prime}\left(\mathbf{a}^{m}\right)\right)^{T} \Delta \mathbf{a}^{I} \\
& +\frac{1}{2}\left(\Delta \mathbf{a}^{I}\right)^{T} \mathbf{S}_{q q, r s}^{\prime \prime}\left(\mathbf{a}^{m}\right) \Delta \mathbf{a}^{I},
\end{aligned}
$$

where

$$
\begin{aligned}
& \mathbf{S}_{q q, r s}^{\prime}\left(\mathbf{a}^{m}\right) \\
& =\left[\frac{\partial S_{q q, r s}\left(\mathbf{a}^{m}\right)}{\partial a_{1}}, \frac{\partial S_{q q, r s}\left(\mathbf{a}^{m}\right)}{\partial a_{2}}, \ldots, \frac{\partial S_{q q, r s}\left(\mathbf{a}^{m}\right)}{\partial a_{N}}\right], \\
& \mathbf{S}_{q q, r s}^{\prime \prime}\left(\mathbf{a}^{m}\right) \\
& =\left[\begin{array}{cccc}
\frac{\partial^{2} S_{q q, r s}\left(\mathbf{a}^{m}\right)}{\partial a_{1} \partial a_{1}} & \frac{\partial^{2} S_{q q, r s}\left(\mathbf{a}^{m}\right)}{\partial a_{1} \partial a_{2}} & \cdots & \frac{\partial^{2} S_{q q, r s}\left(\mathbf{a}^{m}\right)}{\partial a_{1} \partial a_{N}} \\
\frac{\partial^{2} S_{q q, r s}\left(\mathbf{a}^{m}\right)}{\partial a_{2} \partial a_{1}} & \frac{\partial^{2} S_{q q, r s}\left(\mathbf{a}^{m}\right)}{\partial a_{2} \partial a_{2}} & \cdots & \frac{\partial^{2} S_{q q, r s}\left(\mathbf{a}^{m}\right)}{\partial a_{2} \partial a_{N}} \\
\cdots & \cdots & \cdots & \cdots \\
\frac{\partial^{2} S_{q q, r s}\left(\mathbf{a}^{m}\right)}{\partial a_{N} \partial a_{1}} & \frac{\partial^{2} S_{q q, r s}\left(\mathbf{a}^{m}\right)}{\partial a_{N} \partial a_{2}} & \cdots & \frac{\partial^{2} S_{q q, r s}\left(\mathbf{a}^{m}\right)}{\partial a_{N} \partial a_{N}}
\end{array}\right] .
\end{aligned}
$$

If $N$ is large, the computation of the Hessian matrices in (19) and (21) will be intensive. Thus, for the sake of computational efficiency, the nondiagonal elements of the Hessian matrices are neglected, and (21) and (23) are simplified as

$$
\begin{aligned}
E_{j}^{I}= & E_{j}\left(\mathbf{a}^{I}\right) \\
= & E_{j}\left(\mathbf{a}^{m}\right)+\left(\mathbf{E}_{j}^{\prime}\left(\mathbf{a}^{m}\right)\right)^{T} \Delta \mathbf{a}^{I} \\
& +\frac{1}{2}\left(\Delta \mathbf{a}^{I}\right)^{T} \widehat{\mathbf{E}}_{j}^{\prime \prime}\left(\mathbf{a}^{m}\right) \Delta \mathbf{a}^{I}, \\
S_{q q, r s}^{I}= & S_{q q, r s}\left(\mathbf{a}^{I}\right) \\
= & S_{q q, r s}\left(\mathbf{a}^{m}\right)+\left(\mathbf{s}_{q q, r s}^{\prime}\left(\mathbf{a}^{m}\right)\right)^{T} \Delta \mathbf{a}^{I} \\
& +\frac{1}{2}\left(\Delta \mathbf{a}^{I}\right)^{T} \hat{S}_{q q, r s}^{\prime \prime}\left(\mathbf{a}^{m}\right) \Delta \mathbf{a}^{I},
\end{aligned}
$$

where

$$
\begin{aligned}
& \widehat{\mathbf{E}}_{j}^{\prime \prime}\left(\mathbf{a}^{m}\right)=\operatorname{diag}\left[\frac{\partial^{2} E_{j}\left(\mathbf{a}^{m}\right)}{\partial a_{1} \partial a_{1}}, \frac{\partial^{2} E_{j}\left(\mathbf{a}^{m}\right)}{\partial a_{2} \partial a_{2}}, \ldots,\right. \\
& \left.\frac{\partial^{2} E_{j}\left(\mathbf{a}^{m}\right)}{\partial a_{N} \partial a_{N}}\right], \\
& \widehat{\mathbf{S}}_{q q, r s}^{\prime \prime}\left(\mathbf{a}^{m}\right)=\operatorname{diag}\left[\frac{\partial^{2} S_{q q, r s}\left(\mathbf{a}^{m}\right)}{\partial a_{1} \partial a_{1}}, \frac{\partial^{2} S_{q q, r s}\left(\mathbf{a}^{m}\right)}{\partial a_{2} \partial a_{2}}, \ldots,\right. \\
& \left.\quad \frac{\partial^{2} S_{q q, r s}\left(\mathbf{a}^{m}\right)}{\partial a_{N} \partial a_{N}}\right] .
\end{aligned}
$$

4.2. Algorithm for Calculating the Extreme Values of the Expanded Response Variables. For the purpose of calculating the extreme values of the expanded response variables, (25) are rewritten as

$$
\begin{gathered}
E_{j}^{I}=E_{j}\left(\mathbf{a}^{I}\right)=E_{j}\left(\mathbf{a}^{m}\right)+\sum_{i=1}^{N} \Delta E_{j}^{i} \\
S_{q q, r s}^{I}=S_{q q, r s}\left(\mathbf{a}^{I}\right)=S_{q q, r s}\left(\mathbf{a}^{m}\right)+\sum_{i=1}^{N} \Delta S_{q q, r s}^{i},
\end{gathered}
$$

where

$$
\begin{array}{r}
\Delta E_{j}^{i}=\frac{\partial E_{j}\left(\mathbf{a}^{m}\right)}{\partial a_{i}} \Delta \widetilde{a}_{i}+\frac{1}{2} \frac{\partial^{2} E_{j}\left(\mathbf{a}^{m}\right)}{\partial a_{i}^{2}}\left(\Delta \widetilde{a}_{i}\right)^{2}, \\
\Delta \widetilde{a}_{i} \in \Delta a_{i}^{I}=\left[-\Delta a_{i}, \Delta a_{i}\right], \\
\Delta S_{q q, r s}^{i}=\frac{\partial S_{q q, r s}\left(\mathbf{a}^{m}\right)}{\partial a_{i}} \Delta \widetilde{a}_{i}+\frac{1}{2} \frac{\partial^{2} S_{q q, r s}\left(\mathbf{a}^{m}\right)}{\partial a_{i}^{2}}\left(\Delta \widetilde{a}_{i}\right)^{2}, \\
\Delta \widetilde{a}_{i} \in \Delta a_{i}^{I}=\left[-\Delta a_{i}, \Delta a_{i}\right] .
\end{array}
$$

It can be seen from (29) that the expanded equations can be treated as the sum of a series of quadratic functions with 
respect to $\Delta \widetilde{a}_{i}$. Therefore, we can calculate the $\max / \min$ values of $E_{j}^{I}$ and $S_{q q, r s}^{I}$ at the points $-\Delta a_{i}, \Delta a_{i}$, or $a_{i}^{s}$, the values of which can be calculated by

$$
a_{i}^{s}=-\frac{\partial^{2} E_{j}\left(\mathbf{a}^{m}\right) / \partial a_{i}^{2}}{\partial E_{j}\left(\mathbf{a}^{m}\right) / \partial a_{i}}
$$

for (27) and

$$
a_{i}^{s}=-\frac{\partial^{2} S_{q q, r s}\left(\mathbf{a}^{m}\right) / \partial a_{i}^{2}}{\partial S_{q q, r s}\left(\mathbf{a}^{m}\right) / \partial a_{i}}
$$

for (28).

The detailed searching algorithm for the calculation of the extreme values of $\Delta E_{j}^{i}$ and $\Delta S_{q q, r s}^{i}$ is discussed as follows.

If $\Delta a_{i}<\left|a_{i}^{s}\right|$, the $\max / \mathrm{min}$ values of $\Delta E_{j}^{i}\left(\Delta \widetilde{a}_{i}\right)$ and $\Delta S_{q q, r s}^{i}$ can be calculated by

$$
\begin{gathered}
\left(\Delta E_{j}^{i}\right)^{L}=\min \left\{\Delta E_{j}^{i}\left(-\Delta a_{i}\right), \Delta E_{j}^{i}\left(\Delta a_{i}\right)\right\}, \\
\left(\Delta E_{j}^{i}\right)^{R}=\max \left\{\Delta E_{j}^{i}\left(-\Delta a_{i}\right), \Delta E_{j}^{i}\left(\Delta a_{i}\right)\right\}, \\
\left(\Delta S_{q q, r s}^{i}\right)^{L}=\min \left\{\Delta S_{q q, r s}^{i}\left(-\Delta a_{i}\right), \Delta S_{q q, r s}^{i}\left(\Delta a_{i}\right)\right\}, \\
\left(\Delta S_{q q, r s}^{i}\right)^{R}=\max \left\{\Delta S_{q q, r s}^{i}\left(-\Delta a_{i}\right), \Delta S_{q q, r s}^{i}\left(\Delta a_{i}\right)\right\} .
\end{gathered}
$$

If $\Delta a_{i} \geq\left|a_{i}^{s}\right|$, the max $/$ min values of $\Delta E_{j}^{i}\left(\Delta \widetilde{a}_{i}\right)$ and $\Delta S_{q q, r s}^{i}$ can be calculated by

$$
\begin{aligned}
& \left(\Delta E_{j}^{i}\right)^{L}=\min \left\{\Delta E_{j}^{i}\left(-\Delta a_{i}\right), \Delta E_{j}^{i}\left(\Delta a_{i}\right), \Delta E_{j}^{i}\left(a_{i}^{s}\right)\right\}, \\
& \left(\Delta E_{j}^{i}\right)^{R}=\max \left\{\Delta E_{j}^{i}\left(-\Delta a_{i}\right), \Delta E_{j}^{i}\left(\Delta a_{i}\right), \Delta E_{j}^{i}\left(a_{i}^{s}\right)\right\}, \\
& \left(\Delta S_{q q, r s}^{i}\right)^{L} \\
& \quad=\min \left\{\Delta S_{q q, r s}^{i}\left(-\Delta a_{i}\right), \Delta S_{q q, r s}^{i}\left(\Delta a_{i}\right), \Delta S_{q q, r s}^{i}\left(a_{i}^{s}\right)\right\}, \\
& \left(\Delta S_{q q, r s}^{i}\right)^{R} \\
& \quad=\max \left\{\Delta S_{q q, r s}^{i}\left(-\Delta a_{i}\right), \Delta S_{q q, r s}^{i}\left(\Delta a_{i}\right), \Delta S_{q q, r s}^{i}\left(a_{i}^{s}\right)\right\} .
\end{aligned}
$$

Thus, the upper and lower bounds of $E_{j}\left(\mathbf{a}^{I}\right)$ and $S_{q q, r s}\left(\mathbf{a}^{I}\right)$ can be calculated by

$$
\begin{aligned}
E_{j}^{L} & =E_{j}\left(\mathbf{a}^{m}\right)+\sum_{i=1}^{N}\left(\Delta E_{j}^{i}\right)^{L}, \\
E_{j}^{R} & =E_{j}\left(\mathbf{a}^{m}\right)+\sum_{i=1}^{N}\left(\Delta E_{j}^{i}\right)^{R}, \\
S_{q q, r s}^{L} & =S_{q q, r s}\left(\mathbf{a}^{I}\right)=S_{q q, r s}\left(\mathbf{a}^{m}\right)+\sum_{i=1}^{N}\left(\Delta S_{q q, r s}^{i}\right)^{L}, \\
S_{q q, r s}^{R} & =S_{q q, r s}\left(\mathbf{a}^{I}\right)=S_{q q, r s}\left(\mathbf{a}^{m}\right)+\sum_{i=1}^{N}\left(\Delta S_{q q, r s}^{i}\right)^{R} .
\end{aligned}
$$

4.3. Computation of the First- and Second-Order Partial Derivatives of the Interval Response Variables. It can be seen from (27) and (28) that the key to establish the expanded equations is the first- and the second-order partial derivatives of the response variables. To compute the partial derivatives of $E_{j}$ with respect to $a_{i}$, we apply the first- and second-order partial differential operators to (10):

$$
\frac{\partial \mathbf{C}}{\partial a_{i}} \mathbf{E}+\mathbf{C} \frac{\partial \mathbf{E}}{\partial a_{i}}=\frac{\partial \mathbf{P}_{\text {in }}^{\text {ext }}}{\partial a_{i}}+\frac{\partial \mathbf{P}_{\text {in }}}{\partial a_{i}}
$$

$$
\frac{\partial^{2} \mathbf{C}}{\partial a_{i}^{2}} \mathbf{E}+2 \frac{\partial \mathbf{C}}{\partial a_{i}} \frac{\partial \mathbf{E}}{\partial a_{i}}+\mathbf{C} \frac{\partial^{2} \mathbf{E}}{\partial a_{i}^{2}}=\frac{\partial^{2} \mathbf{P}_{\mathrm{in}}^{\text {ext }}}{\partial a_{i}^{2}}+\frac{\partial^{2} \mathbf{P}_{\mathrm{in}}}{\partial a_{i}^{2}}
$$

Given that the term $\mathbf{P}_{\text {in }}$ is independent of $a_{i}$, that is to say, both $\partial \mathbf{P}_{\text {in }} / \partial a_{i}$ and $\partial^{2} \mathbf{P}_{\text {in }} / \partial a_{i}^{2}$ equal zero, thus, the first- and second-order partial derivatives of the energy response vector with respect to $a_{i}$ can be calculated by

$$
\begin{aligned}
\frac{\partial \mathbf{E}}{\partial a_{i}} & =\mathbf{C}^{-1}\left(\frac{\partial \mathbf{P}_{\mathrm{in}}^{\mathrm{ext}}}{\partial a_{i}}-\frac{\partial \mathbf{C}}{\partial a_{i}} \mathbf{E}\right), \\
\frac{\partial^{2} \mathbf{E}}{\partial a_{i}^{2}} & =\mathbf{C}^{-1}\left(\frac{\partial^{2} \mathbf{P}_{\text {in }}^{\mathrm{ext}}}{\partial a_{i}^{2}}-\frac{\partial^{2} \mathbf{C}}{\partial a_{i}^{2}} \mathbf{E}-2 \frac{\partial \mathbf{C}}{\partial a_{i}} \frac{\partial \mathbf{E}}{\partial a_{i}}\right) .
\end{aligned}
$$

By combining (6) (7) and (10), the $j k$ th element of $\partial \mathbf{C} / \partial a_{i}$ and $\partial^{2} \mathbf{C} / \partial a_{i}^{2}$ can be expressed as

$$
\begin{gathered}
\left(\frac{\partial \mathbf{C}}{\partial a_{i}}\right)_{j k}= \begin{cases}\frac{\partial\left(\omega \eta_{d, j}\right)}{\partial a_{i}}+\sum_{k=1, k \neq j}^{N_{s}} \frac{\partial\left(\omega \eta_{j k}\right)}{\partial a_{i}}, & j=k, \\
-\frac{\partial\left(\omega \eta_{j k}\right)}{\partial a_{i}}, & j \neq k,\end{cases} \\
\left(\frac{\partial^{2} \mathbf{C}}{\partial a_{i}^{2}}\right)_{j k}\left(j, k=1,2, \ldots, N_{s}\right), \\
=\left\{\begin{array}{l}
\frac{\partial^{2}\left(\omega \eta_{d, j}\right)}{\partial a_{i}^{2}}+\sum_{k=1, k \neq j}^{N_{s}} \frac{\partial^{2}\left(\omega \eta_{j k}\right)}{\partial a_{i}^{2}}, \quad j=k, \\
-\frac{\partial^{2}\left(\omega \eta_{j k}\right)}{\partial a_{i}^{2}}, \\
\left(j, k=1,2, \ldots, N_{s}\right),
\end{array}\right.
\end{gathered}
$$


where

$$
\begin{aligned}
& \frac{\partial\left(\omega \eta_{d, j}\right)}{\partial a_{i}}=\left(\frac{2 \alpha_{j}}{\pi n_{j}}\right) \\
& \cdot \sum_{r s} \frac{\partial\left(\operatorname{Im}\left\{D_{d, r s}\right\}\left(\mathbf{D}_{\mathrm{tot}}^{-1} \operatorname{Im}\left\{\mathbf{D}_{\mathrm{dir}}^{(j)}\right\} \mathbf{D}_{\mathrm{tot}}^{-H}\right)_{r s}\right)}{\partial a_{i}}, \\
& \frac{\partial\left(\omega \eta_{j k}\right)}{\partial a_{i}}=\left(\frac{2 \alpha_{k}}{\pi n_{j}}\right) \\
& \cdot \sum_{r s} \operatorname{Im}\left\{D_{\text {dir }, r s}^{(j)}\right\} \frac{\partial\left(\mathbf{D}_{\text {tot }}^{-1} \operatorname{Im}\left\{\mathbf{D}_{\text {dir }}^{(k)}\right\} \mathbf{D}_{\text {tot }}^{-H}\right)_{r s}}{\partial a_{i}}, \\
& \frac{\partial^{2}\left(\omega \eta_{d, j}\right)}{\partial a_{i}^{2}}=\left(\frac{2 \alpha_{j}}{\pi n_{j}}\right) \\
& \cdot \sum_{r s} \frac{\partial^{2}\left(\operatorname{Im}\left\{D_{d, r s}\right\}\left(\mathbf{D}_{\mathrm{tot}}^{-1} \operatorname{Im}\left\{\mathbf{D}_{\mathrm{dir}}^{(j)}\right\} \mathbf{D}_{\mathrm{tot}}^{-H}\right)_{r s}\right)}{\partial a_{i}^{2}}, \\
& \frac{\partial^{2}\left(\omega \eta_{j k}\right)}{\partial a_{i}^{2}}=\left(\frac{2 \alpha_{k}}{\pi n_{j}}\right) \\
& \cdot \sum_{r s} \operatorname{Im}\left\{D_{\mathrm{dir}, r s}^{(j)}\right\} \frac{\partial^{2}\left(\mathbf{D}_{\mathrm{tot}}^{-1} \operatorname{Im}\left\{\mathbf{D}_{\mathrm{dir}}^{(k)}\right\} \mathbf{D}_{\mathrm{tot}}^{-H}\right)_{r s}}{\partial a_{i}^{2}} .
\end{aligned}
$$

From (8), we can see that the $j$ th element of $\partial \mathbf{P}_{\text {in }}^{\text {ext }} / \partial a_{i}$ and $\partial^{2} \mathbf{P}_{\text {in }}^{\text {ext }} / \partial a_{i}^{2}$ can be expressed as

$$
\begin{aligned}
\frac{\partial P_{\mathrm{in}, j}^{\mathrm{ext}}}{\partial a_{i}} & =\left(\frac{\omega}{2}\right) \sum_{r s} \operatorname{Im}\left\{D_{\mathrm{dir}, r s}^{(j)}\right\} \frac{\partial\left(\mathbf{D}_{\mathrm{tot}}^{-1} \mathbf{S}_{f f} \mathbf{D}_{\mathrm{tot}}^{-H}\right)_{r s}}{\partial a_{i}} \\
\frac{\partial^{2} P_{\mathrm{in}, j}^{\mathrm{ext}}}{\partial a_{i}^{2}} & =\left(\frac{\omega}{2}\right) \sum_{r s} \operatorname{Im}\left\{D_{\mathrm{dir}, r s}^{(j)}\right\} \frac{\partial^{2}\left(\mathbf{D}_{\mathrm{tot}}^{-1} \mathbf{S}_{f f} \mathbf{D}_{\mathrm{tot}}^{-H}\right)_{r s}}{\partial a_{i}^{2}} .
\end{aligned}
$$

It can be seen from (38) (39) that the key to calculating the first- and the second-order partial derivatives of the energy response vector is the first- and second-order partial derivatives of $\mathbf{D}_{\text {tot }}^{-1}$, which can be calculated by

$$
\begin{aligned}
\frac{\partial \mathbf{D}_{\text {tot }}^{-1}}{\partial a_{i}} & =-\mathbf{D}_{\text {tot }}^{-1} \frac{\partial \mathbf{D}_{\text {tot }}}{\partial a_{i}} \mathbf{D}_{\text {tot }}^{-1} \\
\frac{\partial^{2} \mathbf{D}_{\text {tot }}^{-1}}{\partial a_{i}^{2}} & =-\left(\frac{\partial \mathbf{D}_{\text {tot }}^{-1}}{\partial a_{i}} \frac{\partial \mathbf{D}_{\text {tot }}}{\partial a_{i}} \mathbf{D}_{\text {tot }}^{-1}+\mathbf{D}_{\text {tot }}^{-1} \frac{\partial^{2} \mathbf{D}_{\text {tot }}}{\partial a_{i}^{2}} \mathbf{D}_{\text {tot }}^{-1}\right. \\
+\mathbf{D}_{\text {tot }}^{-1} & \left.\frac{\partial \mathbf{D}_{\text {tot }}}{\partial a_{i}} \frac{\partial \mathbf{D}_{\text {tot }}^{-1}}{\partial a_{i}}\right) .
\end{aligned}
$$

Thus, by submitting the mean value vector $\mathbf{a}_{m}$ into (36), the partial derivatives of $E_{j}$ with respect to $a_{i}$ can be written as

$$
\begin{aligned}
& \frac{\partial E_{j}\left(\mathbf{a}_{m}\right)}{\partial a_{i}}=\left(\mathbf { C } ^ { - 1 } ( \mathbf { a } _ { m } ) \left(\frac{\partial \mathbf{P}_{\mathrm{in}}^{\text {ext }}\left(\mathbf{a}_{m}\right)}{\partial a_{i}}\right.\right. \\
& \left.\left.-\frac{\partial \mathbf{C}\left(\mathbf{a}_{m}\right)}{\partial a_{i}} \mathbf{E}\left(\mathbf{a}_{m}\right)\right)\right)_{j} \\
& \frac{\partial^{2} E_{j}\left(\mathbf{a}_{m}\right)}{\partial a_{i}^{2}}=\left(\mathbf { C } ^ { - 1 } ( \mathbf { a } _ { m } ) \left(\frac{\partial^{2} \mathbf{P}_{\mathrm{in}}^{\text {ext }}\left(\mathbf{a}_{m}\right)}{\partial a_{i}^{2}}\right.\right. \\
& \left.\left.-\frac{\partial^{2} \mathbf{C}\left(\mathbf{a}_{m}\right)}{\partial a_{i}^{2}} \mathbf{E}\left(\mathbf{a}_{m}\right)-2 \frac{\partial \mathbf{C}\left(\mathbf{a}_{m}\right)}{\partial a_{i}} \frac{\partial \mathbf{E}\left(\mathbf{a}_{m}\right)}{\partial a_{i}}\right)\right)_{j} .
\end{aligned}
$$

Similarly, by applying the first- and second-order partial differential operators to (13), we get

$$
\begin{aligned}
& \frac{\partial \mathbf{S}_{q q}\left(\mathbf{a}_{m}\right)}{\partial a_{i}} \\
& =\left.\frac{\partial\left(\mathbf{D}_{\mathrm{tot}}^{-1} \mathbf{S}_{f f} \mathbf{D}_{\mathrm{tot}}^{-H}\right)}{\partial a_{i}}\right|_{\mathbf{a}=\mathbf{a}_{m}} \\
& \quad+\left.\sum_{j} \frac{4 \alpha_{j}}{\pi \omega n_{j}} \frac{\partial\left(\mathbf{D}_{\mathrm{tot}}^{-1} E_{j} \operatorname{Im}\left\{\mathbf{D}_{\mathrm{dir}}^{(j)}\right\} \mathbf{D}_{\mathrm{tot}}^{-H}\right)}{\partial a_{i}}\right|_{\mathbf{a}=\mathbf{a}_{m}}, \\
& \frac{\partial^{2} \mathbf{S}_{q q}\left(\mathbf{a}_{m}\right)}{\partial a_{i}^{2}} \\
& =\left.\frac{\partial^{2}\left(\mathbf{D}_{\mathrm{tot}}^{-1} \mathbf{S}_{f f} \mathbf{D}_{\mathrm{tot}}^{-H}\right)}{\partial a_{i}^{2}}\right|_{\mathbf{a}=\mathbf{a}_{m}}, \\
& \quad+\left.\sum_{j} \frac{4 \alpha_{j}}{\pi \omega n_{j}} \frac{\partial^{2}\left(\mathbf{D}_{\mathrm{tot}}^{-1} E_{j} \operatorname{Im}\left\{\mathbf{D}_{\mathrm{dir}}^{(j)}\right\} \mathbf{D}_{\mathrm{tot}}^{-H}\right)}{\partial a_{i}^{2}}\right|_{\mathbf{a}=\mathbf{a}_{m}} .
\end{aligned}
$$

It can be seen from (43) that the key to calculating the first- and the second-order partial derivatives of the response cross-spectrum matrix is the first- and second-order partial derivatives of $\mathbf{D}_{\text {tot }}^{-1}$ and $E_{j}$, and they can be calculated by 
combining (40) (42). Thus, the $r s$ th element of $\partial \mathbf{S}_{q q}\left(\mathbf{a}_{m}\right) / \partial a_{i}$ and $\partial^{2} \mathbf{S}_{q q}\left(\mathbf{a}_{m}\right) / \partial a_{i}^{2}$ can be expressed as

$$
\begin{aligned}
& \frac{\partial S_{q q, r s}\left(\mathbf{a}^{m}\right)}{\partial a_{i}}=\left(\left.\frac{\partial\left(\mathbf{D}_{\mathrm{tot}}^{-1} \mathbf{S}_{f f} \mathbf{D}_{\mathrm{tot}}^{-H}\right)}{\partial a_{i}}\right|_{\mathbf{a}=\mathbf{a}_{m}}\right. \\
& \left.\quad+\left.\sum_{j} \frac{4 \alpha_{j}}{\pi \omega n_{j}} \frac{\partial\left(\mathbf{D}_{\mathrm{tot}}^{-1} E_{j} \operatorname{Im}\left\{\mathbf{D}_{\mathrm{dir}}^{(j)}\right\} \mathbf{D}_{\mathrm{tot}}^{-H}\right)}{\partial a_{i}}\right|_{\mathbf{a}=\mathbf{a}_{m}}\right)_{r s}, \\
& \frac{\partial^{2} S_{q q, r s}\left(\mathbf{a}^{m}\right)}{\partial a_{i}^{2}}=\left(\left.\frac{\partial^{2}\left(\mathbf{D}_{\mathrm{tot}}^{-1} \mathbf{S}_{f f} \mathbf{D}_{\mathrm{tot}}^{-H}\right)}{\partial a_{i}^{2}}\right|_{\mathbf{a}=\mathbf{a}_{m}}\right. \\
& \left.\quad+\left.\sum_{j} \frac{4 \alpha_{j}}{\pi \omega n_{j}} \frac{\partial^{2}\left(\mathbf{D}_{\mathrm{tot}}^{-1} E_{j} \operatorname{Im}\left\{\mathbf{D}_{\mathrm{dir}}^{(j)}\right\} \mathbf{D}_{\mathrm{tot}}^{-H}\right)}{\partial a_{i}^{2}}\right|_{\mathbf{a}=\mathbf{a}_{m}}\right)_{r s} .
\end{aligned}
$$

4.4. The Procedure of the SIPFEM/SEA Method for the Midfrequency Analysis of Built-Up Structure Systems with Interval Parameters. The main steps of SIPFEM/SEA for the midfrequency analysis of built-up structure systems with interval parameters are summarized as follows.

Step 1. Partition the built-up structure system into a combination of the master system and the subsystem and establish the equations of FE-SEA (equations (5) and (13)).

Step 2. Introduce interval parametric uncertainty into the master system within the hybrid FE-SEA framework and establish the interval equations (equations (15) and (16)).

Step 3. Expand the interval response variables $\left(E_{j}^{I}\right.$ and $\left.\mathbf{S}_{q q}^{I}\right)$ of the built-up structure system with the second-order Taylor series at the mean values of interval parameters, and for the sake of simplicity and efficiency, the nondiagonal elements of the Hessian matrices are neglected (equations (25) (26)).

Step 4. Calculate the first- and second-order partial derivatives of the interval response variables (equations (41) (43)).

Step 5. Search the target positions of interval parameters that maximize or minimize the objective functions, and calculate the bounds of the expanded responses by submitting the target values into the objective functions (equations (32) (34)).

\section{The Formulation of the Subinterval Perturbation Method Based on SIPFEM/SEA for the Midfrequency Analysis of Built-Up Structure Systems with Interval Parameters}

Because of the neglect of the higher order terms of Taylor series, SIPFEM/SEA is limited to the midfrequency analysis of the built-up structure systems with small interval parametric uncertainty. In order to predict the response of the built-up structure systems with large interval parametric uncertainty, the subinterval perturbation method based on SIPFEM/SEA is introduced in this section.

By dividing the large interval parameters $a_{i}^{I}(i=$ $1,2, \ldots, N)$ into $L_{i}$ small subintervals, one can get

$$
\begin{aligned}
a_{r_{i}, i}^{I}=\left[a_{i}^{L}+\frac{2\left(r_{i}-1\right) \Delta a_{i}}{L_{i}}, a_{i}^{L}+\frac{2 r_{i} \Delta a_{i}}{L_{i}}\right] & \\
r_{i} & =1,2, \ldots, L_{i},
\end{aligned}
$$

where $a_{r_{i}, i}^{I}$ is the $r_{i}$ th subinterval of the $i$ th interval parameter $a_{i}^{I}$. According to permutation and combination theory, we can see that the number of the subinterval combinations is $\prod_{i=1}^{N} L_{i}$, and each subinterval combination can be expressed as

$$
\begin{aligned}
& \mathbf{a}_{r_{1} r_{2} \cdots r_{N}}^{I}=\left(a_{r_{1}, 1}^{I}, a_{r_{2}, 2}^{I}, \ldots, a_{r_{N}, N}^{I}\right), \\
& \quad\left(r_{i}=1,2, \ldots, L_{i}, i=1,2, \ldots, N\right) .
\end{aligned}
$$

By applying the SIPFEM/SEA method to each subinterval combination, the corresponding subintervals of the response variables can be obtained and expressed as

$$
\begin{aligned}
E_{j, r_{1} r_{2} \cdots r_{N}}^{I}= & {\left[E_{j, r_{1} r_{2} \cdots r_{N}}^{L}, E_{j, r_{1} r_{2} \cdots r_{N}}^{R}\right], } \\
\mathbf{S}_{q q, r_{1} r_{2} \cdots r_{N}}^{I}= & {\left[\mathbf{S}_{q q, r_{1} r_{2} \cdots r_{N}}^{L}, \mathbf{S}_{q q, r_{1} r_{2} \cdots r_{N}}^{R}\right], } \\
& \left(r_{i}=1,2, \ldots, L_{i}, i=1,2, \ldots, N\right) .
\end{aligned}
$$

By assembling the subintervals of the response variables with the interval union operation, the global intervals of the response variables can be obtained and expressed as

$$
\begin{aligned}
& E_{j}\left(\mathbf{a}^{I}\right)=\left[\min \left(E_{j, r_{1} r_{2} \cdots r_{N}}^{L}\right), \max \left(E_{j, r_{1} r_{2} \cdots r_{N}}^{R}\right)\right], \\
& \mathbf{S}_{q q}\left(\mathbf{a}^{I}\right)=\left[\min \left(\mathbf{S}_{q q, r_{1} r_{2} \cdots r_{N}}^{L}\right), \max \left(\mathbf{S}_{q q, r_{1} r_{2} \cdots r_{N}}^{R}\right)\right], \\
& \quad\left(r_{i}=1,2, \ldots, L_{i}, i=1,2, \ldots, N\right) .
\end{aligned}
$$

From the convergence condition of perturbation theory, we can see that if the number of the subintervals for each parameter is sufficiently large, the bounds of the response variables of the built-up structure systems with large interval parametric uncertainty will be predicted accurately.

\section{Numerical Examples}

6.1. An Oscillator-Plate System. Figure 1 shows an oscillatorplate system in which the oscillator is attached to the simply supported plate. The dimensions of the plate are $2.1 \mathrm{~m} \times$ $1.9 \mathrm{~m} \times 1.25 \mathrm{~mm}$, Young's modulus is $E_{p}=7.2 \times 10^{4} \mathrm{MPa}$, the density is $\rho_{p}=2800 \mathrm{~kg} / \mathrm{m}^{3}$, Poisson's ratio is $\nu=0.3$, and the modal density is $n_{p}=1.05$ modes $/ \mathrm{Hz}$. The oscillator consists of a spring and a mass, and it is attached at the point $(0.882$, 0.772). The spring is fixed at the other end and a vertical unit force $F$ is applied to the mass. The stiffness of the spring is expressed as $k$, and the mass value is expressed as $m$. The damping loss factors of the plate and the oscillator are both $\eta=0.01$. 


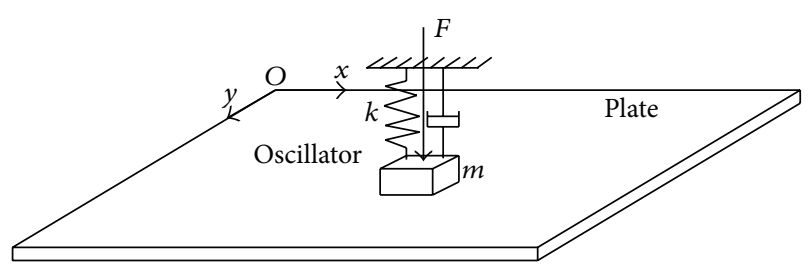

Figure 1: The oscillator-plate system.

To describe the system via the FE-SEA method, the oscillator is modeled as the FE component, and the plate is modeled as the SEA subsystem. Considering the parametric uncertainty of the FE component, the stiffness of the spring $k$ and the mass value $m$ are set as the interval parameters. By introducing the uncertainty level $\alpha$, the intervals of the spring $k$ and the mass value $m$ can be expressed as

$$
\begin{aligned}
& k^{I}=k_{m}[1-\alpha, 1+\alpha], \\
& m^{I}=m_{m}[1-\alpha, 1+\alpha],
\end{aligned}
$$

where the mean values of $k$ and $m$ are $k_{m}=3.2 \times 10^{6} \mathrm{~N} / \mathrm{m}$, $m_{m}=2 \mathrm{~kg}$.

To investigate the accuracy of the proposed SIPFEM/SEA method for predicting the response of the built-up structure system with interval parameters, we calculate the lower and upper bounds of the plate energy and the autospectra of the mass displacements. Benchmark results are made by the Monte Carlo simulations of the hybrid FE/SEA model with 10,000 samples, and all of the results for this oscillator-plate system are obtained by using MATLAB R2014a on a $3.60 \mathrm{GHz}$ Intel(R) Core (TM) CPU i7-4790. Figures 2 and 3 show the bounds of the plate energy and the autospectra of the mass displacements for frequencies $100 \sim 300 \mathrm{~Hz}$, respectively. The considered uncertainty levels are $\alpha=0.01,0.02,0.03$, and 0.04. From Figures 3 and 4, we can see that there is an excellent agreement between the results yielded by the SIPFEM/SEA method and by the Monte Carlo simulation of the hybrid FE/SEA model when the uncertainty level $\alpha$ is not more than 0.02 , and there is a significant deviation between the results yielded by the two methods when the uncertainty level $\alpha$ is increased to 0.04 . Due to the effect of the inherent flaw of the perturbation method, the bounds calculated by SIPFEM/SEA at the neighborhood of the peaks corresponding to the resonance frequencies of the oscillator are unreliable and valueless.

To investigate the accuracy of the proposed SIPFEM/SEA method for predicting the response of the built-up structure system with interval parameters more clearly, the relative errors of the bounds of the plate energy and the autospectra of the mass displacements obtained by SIPFEM/SEA for $\alpha=$ $0.01,0.02,0.03$, and 0.04 are calculated and listed in Tables 1 and 2; the considered frequencies are $f=170 \mathrm{~Hz}$ and $f=230 \mathrm{~Hz}$, respectively. It can be seen from Tables 1 and 2 that the relative errors of SIPFEM/SEA increase gradually with the increase of the uncertainty level $\alpha$. From Figures 1 and 2 and Tables 1 and 2, we can conclude that the proposed SIPFEM/SEA method is very accurate for predicting the response of the built-up structure system with small interval parametric uncertainty, and when the uncertainty level gets larger, the accuracy of it decreases and unreliable results will be obtained. This is mainly because of the neglect of the higher order terms of Taylor series, which may bring unpredictable and uncontrollable effect on the results.

To extend SIPFEM/SEA to the midfrequency analysis of the built-up structure system with large interval parameters, the subinterval perturbation method is introduced based on SIPFEM/SEA(S-SIPFEM/SEA). To illustrate the accuracy of S-SIPFEM/SEA, the uncertainty level $\alpha$ is set as 0.08 ; other parameters are kept the same. Figures 4 and 5 show the bounds of the plate energy and the autospectra of the mass displacements for $\alpha=0.08$, respectively. The considered numbers of subintervals for each parameter are one, two, four, and eight. It can be seen from Figures 4 and 5 that the bounds of the plate energy and the autospectra yielded by S-SIPFEM/SEA match the reference bounds more closely with the increase of the number of the subintervals, and the bounds yielded by S-SIPFEM/SEA with eight subintervals match the reference bounds perfectly. The relative errors of the bounds yielded by S-SIPFEM/SEA with different subinterval numbers are shown in Tables 3 and 4, and the considered frequencies are $f=170 \mathrm{~Hz}$ and $f=230 \mathrm{~Hz}$, respectively. From Tables 3 and 4, we can see that the relative errors of S-SIPFEM/SEA get smaller with the increase of the number of subintervals. Therefore, we can conclude that S-SIPFEM/SEA will be accurate for the midfrequency analysis of the built-up structure system with large interval parameters if the number of the subintervals is sufficiently large.

Execution times of the S-SIPFEM/SEA with eight subintervals and the Monte Carlo simulations of the hybrid FE/SEA model for calculating the plate energy are shown in Table 5; we can see that the S-SIPFEM/SEA is much more efficient than the Monte Carlo simulation of the hybrid FE/SEA model.

6.2. A Beam-Plate System. Beam-plate systems are typical built-up structures in practical engineering, and the beams are usually much stiffer than the plates [39]. Figure 6 depicts a beam-plate system in which a plate and a beam are coupled via three point connections. The plate is rectangular with dimensions of $2 \mathrm{~m} \times 0.8 \mathrm{~m} \times 5 \mathrm{~mm}$, and Young's modulus of it is $E_{p}=2.1 \times 10^{5} \mathrm{MPa}$, the density is $\rho_{p}=7850 \mathrm{~kg} / \mathrm{m}^{3}$, Poisson's ratio is $\nu_{p}=0.3$, and the modal density is $n_{p}=$ 0.102 modes $/ \mathrm{Hz}$. The beam with a length of $l_{b}=2 \mathrm{~m}$ is simply supported at both ends, and the cross section of it is rectangular with dimensions of $0.03 \mathrm{~m} \times 0.04 \mathrm{~m}$; Poisson's ratio of the beam is $\nu_{b}=0.3$; Young's modulus and the density of it are expressed as $E_{b}$ and $\rho_{b}$, respectively. The damping loss factors of the beam and the plate are both $\eta=0.01$. The coupling points are located along the beam length at $0.27 l_{b}$, $0.47 l_{b}$, and $0.69 l_{b}$, and a force $F$ is vertically exerted at $0.37 l_{b}$ of the beam. In order to employ FE-SEA to model this system, the beam is set as the master system and modeled by using FEM with 200 beam elements; the plate is set as the subsystem and modeled by SEA. 
TABLE 1: The relative errors of the bounds of the plate energy and the autospectrum of the mass displacements for different uncertainty levels $(f=170 \mathrm{~Hz})$.

\begin{tabular}{cccccccc}
\hline & \multirow{2}{*}{ Uncertainty level $\alpha$} & \multicolumn{3}{c}{ Lower bounds } & \multicolumn{2}{c}{ Upper bounds } \\
& & MCS & SIPFEM/SEA & Relative errors (\%) & MCS & SIPFEM/SEA & Relative errors (\%) \\
\hline \multirow{3}{*}{$E(\mathrm{~J})$} & 0.01 & $4.15 E-06$ & $4.12 E-06$ & 0.72 & $5.50 E-06$ & $5.46 E-06$ & 0.73 \\
& 0.02 & $3.65 E-06$ & $3.55 E-06$ & 2.74 & $6.41 E-06$ & $6.25 E-06$ & 2.50 \\
& 0.03 & $3.25 E-06$ & $3.06 E-06$ & 5.85 & $7.61 E-06$ & $7.10 E-06$ & 6.70 \\
& 0.04 & $2.90 E-06$ & $2.64 E-06$ & 8.97 & $9.11 E-06$ & $8.03 E-06$ & 11.86 \\
\hline \multirow{3}{*}{$S_{q q}\left(\mathrm{~m}^{2}\right)$} & 0.01 & $1.41 E-12$ & $1.40 E-12$ & 0.71 & $1.88 E-12$ & $1.86 E-12$ & 1.06 \\
& 0.02 & $1.24 E-12$ & $1.21 E-12$ & 2.42 & $2.20 E-12$ & $2.13 E-12$ & 3.18 \\
& 0.03 & $1.10 E-12$ & $1.04 E-12$ & 5.45 & $2.59 E-12$ & $2.42 E-12$ & 6.56 \\
& 0.04 & $9.91 E-13$ & $8.98 E-13$ & 9.38 & $3.16 E-12$ & $2.74 E-12$ \\
\hline
\end{tabular}

TABLE 2: The relative errors of the bounds of the plate energy and the autospectrum of the mass displacements for different uncertainty levels $(f=230 \mathrm{~Hz})$.

\begin{tabular}{cccccccc}
\hline & \multirow{2}{*}{ Uncertainty level $\alpha$} & \multicolumn{3}{c}{ Lower bounds } & \multicolumn{2}{c}{ Upper bounds } \\
& & MCS & SIPFEM/SEA & Relative errors (\%) & MCS & SIPFEM/SEA & Relative errors (\%) \\
\hline \multirow{3}{*}{$E(\mathrm{~J})$} & 0.01 & $4.95 E-06$ & $4.89 E-06$ & 1.21 & $7.03 E-06$ & $6.96 E-06$ & 1.00 \\
& 0.02 & $4.26 E-06$ & $4.06 E-06$ & 4.69 & $8.59 E-06$ & $8.20 E-06$ & 4.54 \\
& 0.03 & $3.67 E-06$ & $3.37 E-06$ & 8.17 & $1.08 E-05$ & $9.58 E-06$ & 11.30 \\
\hline \multirow{4}{*}{$S_{q q}\left(\mathrm{~m}^{2}\right)$} & 0.04 & $3.21 E-06$ & $2.82 E-06$ & 12.15 & $1.38 E-05$ & $1.11 E-05$ & 19.57 \\
& 0.01 & $1.31 E-12$ & $1.29 E-12$ & 1.53 & $1.87 E-12$ & $1.85 E-12$ & 1.07 \\
& 0.02 & $1.13 E-12$ & $1.07 E-12$ & 5.31 & $2.28 E-12$ & $2.18 E-12$ & 4.39 \\
& 0.03 & $9.71 E-13$ & $8.91 E-13$ & 8.24 & $2.83 E-12$ & $2.55 E-12$ & 9.89 \\
\hline
\end{tabular}

TABLE 3: The relative errors of the bounds of the plate energy and the autospectra of the mass displacements yielded by S-SIPFEM/SEA with different subinterval numbers $(f=170 \mathrm{~Hz})$.

\begin{tabular}{|c|c|c|c|c|c|c|c|}
\hline & \multirow{2}{*}{ The number of subintervals } & \multicolumn{3}{|c|}{ Lower bounds } & \multicolumn{3}{|c|}{ Upper bounds } \\
\hline & & MCS & S-SIPFEM/SEA & Relative errors (\%) & MCS & S-SIPFEM/SEA & Relative errors (\%) \\
\hline \multirow{4}{*}{$E(\mathrm{~J})$} & 1 & \multirow{4}{*}{$1.57 E-06$} & $1.34 E-06$ & 14.65 & \multirow{4}{*}{$1.20 E-05$} & $7.70 E-06$ & 35.83 \\
\hline & 2 & & $1.48 E-06$ & 5.73 & & $1.02 E-05$ & 15.00 \\
\hline & 4 & & $1.53 E-06$ & 2.55 & & $1.14 E-05$ & 5.00 \\
\hline & 8 & & $1.55 E-06$ & 1.27 & & $1.18 E-05$ & 1.67 \\
\hline \multirow{4}{*}{$S_{q q}\left(\mathrm{~m}^{2}\right)$} & 1 & \multirow{4}{*}{$5.49 E-13$} & $4.68 E-13$ & 14.75 & \multirow{4}{*}{$4.22 E-12$} & $2.71 E-12$ & 35.78 \\
\hline & 2 & & $5.18 E-13$ & 5.65 & & $3.58 E-12$ & 15.17 \\
\hline & 4 & & $5.36 E-13$ & 2.37 & & $4.02 E-12$ & 4.74 \\
\hline & 8 & & $5.40 E-13$ & 1.64 & & $4.18 E-12$ & 0.95 \\
\hline
\end{tabular}

TABLE 4: The relative errors of the bounds of the plate energy and the autospectra of the mass displacements yielded by S-SIPFEM/SEA with different subinterval numbers $(f=230 \mathrm{~Hz})$.

\begin{tabular}{|c|c|c|c|c|c|c|c|}
\hline & \multirow{2}{*}{ The number of subintervals } & \multicolumn{3}{|c|}{ Lower bounds } & \multicolumn{3}{|c|}{ Upper bounds } \\
\hline & & MCS & S-SIPFEM/SEA & Relative errors (\%) & MCS & S-SIPFEM/SEA & Relative errors (\%) \\
\hline \multirow{4}{*}{$E(\mathrm{~J})$} & 1 & \multirow{4}{*}{$1.58 E-06$} & $1.39 E-06$ & 12.03 & \multirow{4}{*}{$2.22 E-05$} & $1.09 E-05$ & 50.90 \\
\hline & 2 & & $1.48 E-06$ & 6.33 & & $1.71 E-05$ & 22.97 \\
\hline & 4 & & $1.55 E-06$ & 1.90 & & $2.11 E-05$ & 4.95 \\
\hline & 8 & & $1.57 E-06$ & 0.63 & & $2.29 E-05$ & 3.15 \\
\hline \multirow{4}{*}{$S_{q q}\left(\mathrm{~m}^{2}\right)$} & 1 & \multirow{4}{*}{$4.09 E-13$} & $3.61 E-13$ & 11.74 & \multirow{4}{*}{$6.15 E-12$} & $2.85 E-12$ & 53.66 \\
\hline & 2 & & $3.81 E-13$ & 6.85 & & $4.48 E-12$ & 27.15 \\
\hline & 4 & & $4.00 E-13$ & 2.20 & & $5.57 E-12$ & 9.43 \\
\hline & 8 & & $4.05 E-13$ & 0.98 & & $6.06 E-12$ & 1.46 \\
\hline
\end{tabular}




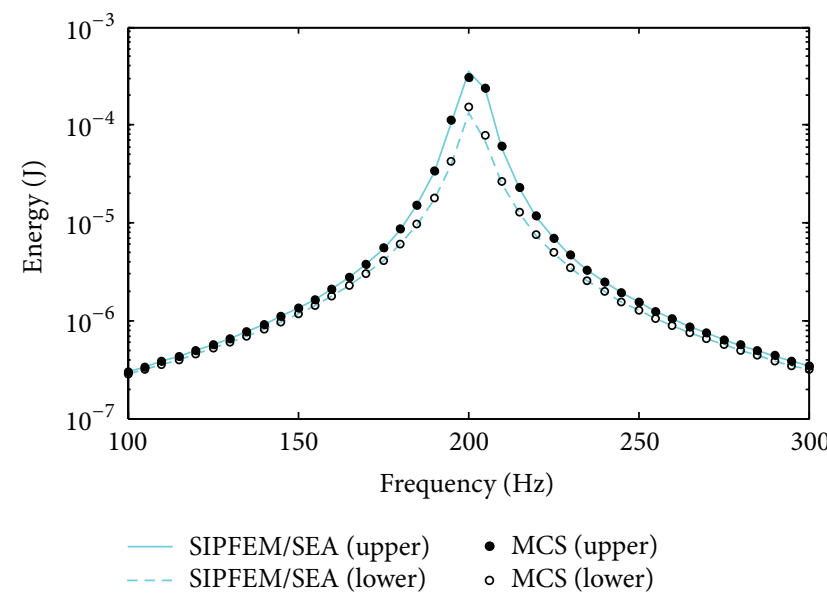

(a)

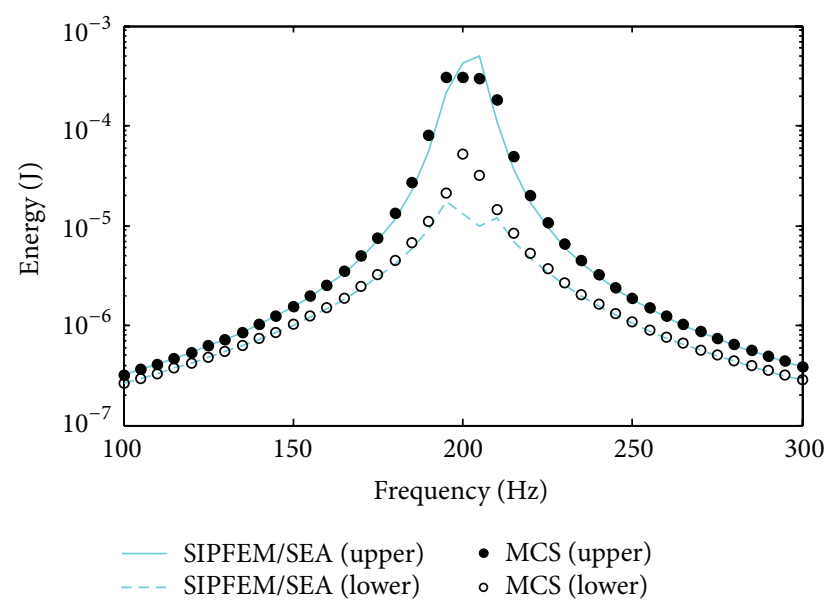

(c)

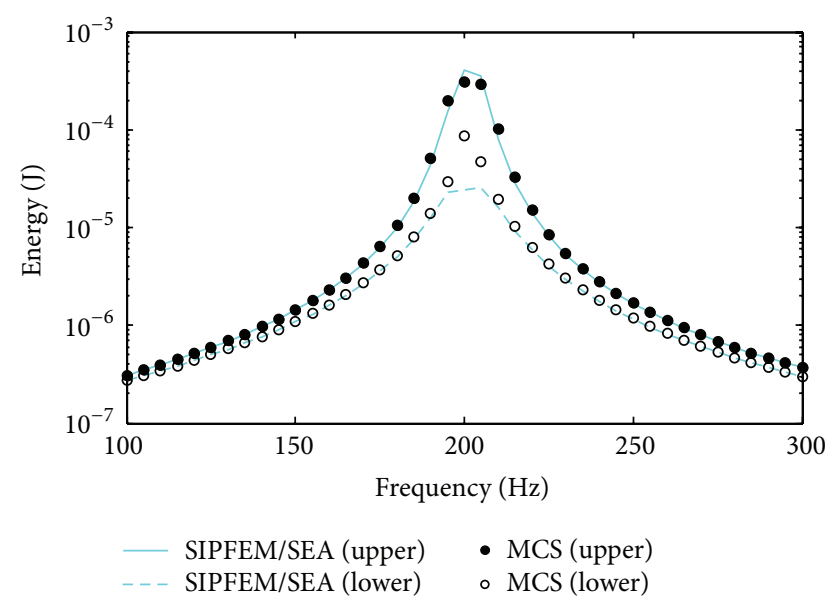

(b)

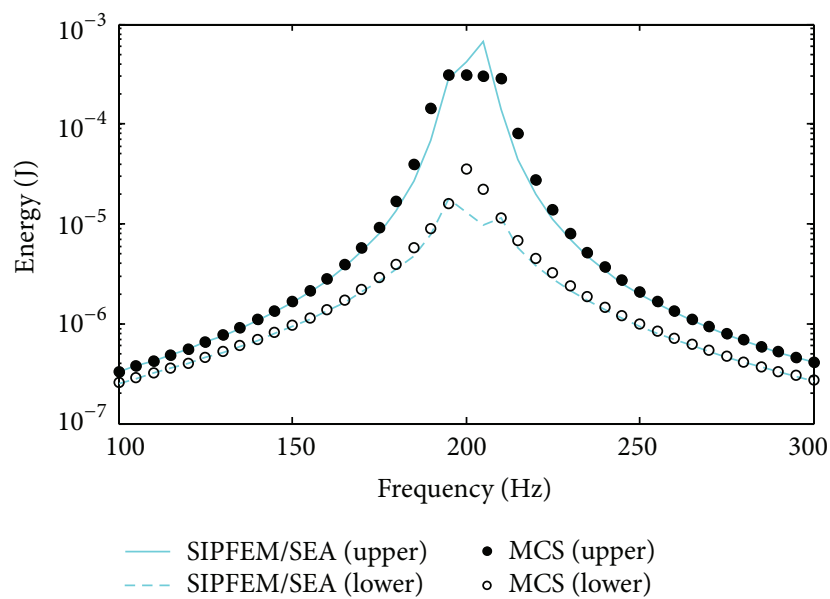

(d)

FIGURE 2: The bounds of the plate energy: (a) $\alpha=0.01$; (b) $\alpha=0.02$; (c) $\alpha=0.03$; (d) $\alpha=0.04$.

TABLE 5: Execution times of S-SIPFEM/SEA and the Monte Carlo simulation of the hybrid FE/SEA model.

\begin{tabular}{lcc}
\hline & MCS & S-SIPFEM/SEA \\
\hline Execution time(s) & 9.22 & 0.19 \\
\hline
\end{tabular}

In this numerical example, Young's modulus $E_{b}$ and the density $\rho_{b}$ of the beam and the force $F$ are considered as interval parameters, and the intervals of them can be expressed by using the uncertainty level $\alpha$ :

$$
\begin{aligned}
& E_{b}^{I}=E_{b, m}[1-\alpha, 1+\alpha], \\
& \rho_{b}^{I}=\rho_{b, m}[1-\alpha, 1+\alpha], \\
& F^{I}=F_{m}[1-\alpha, 1+\alpha],
\end{aligned}
$$

where the mean values of $E_{b}, \rho_{b}$, and $F$ are $E_{b, m}=2.1 \times$ $10^{5} \mathrm{Mpa}, \rho_{b, m}=7850 \mathrm{~kg}$, and $F_{m}=1 \mathrm{~N}$, respectively.

All simulations of the beam-plate system are carried out by using MATLAB R2014a on a $3.60 \mathrm{GHz}$ Intel(R) Core (TM) CPU i7-4790. To illustrate the proposed SIPFEM/SEA method, the bounds of the plate energy and the autospectra of the driving point displacements are calculated, and benchmark results are obtained by the Monte Carlo simulations of the hybrid FE/SEA model with 10,000 samples. The bounds of the plate energy and the autospectra of the driving point displacements in the frequency band $f=200 \sim 600 \mathrm{~Hz}$ are shown in Figures 7 and 8; the considered uncertainty levels of the interval parameters are $\alpha=0.01,0.02,0.03$, and 0.04 , respectively. The relative errors of the bounds of the plate energy and the autospectra of the driving point displacements with different uncertainty levels are shown in Tables 6 and 7; the considered frequencies are $f=330 \mathrm{~Hz}$ and $f=440 \mathrm{~Hz}$. From Figures 7 and 8 and Tables 6 and 7, we can conclude that the accuracy of SIPFEM/SEA for midfrequency analysis of built-up structure systems with interval parameters of small uncertainty levels is excellent, and it will decrease gradually with the increase of the uncertainty levels. If the uncertainty level increases to a certain value, unreliable results will be yielded by SIPFEM/SEA.

To illustrate the effectiveness of S-SIPFEM/SEA for the analysis with large uncertainty level, we take the uncertainty level $\alpha=0.04$ as an example. Figures 9 and 10 show 


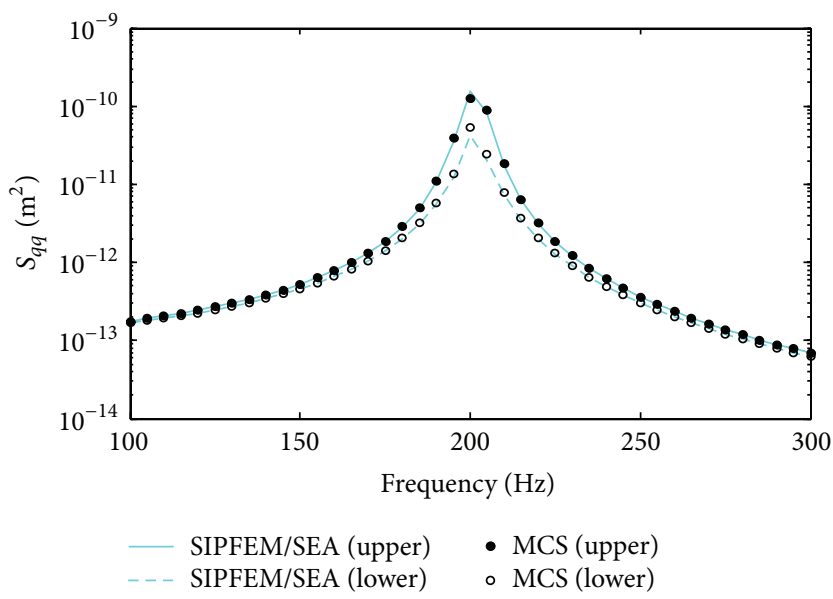

(a)

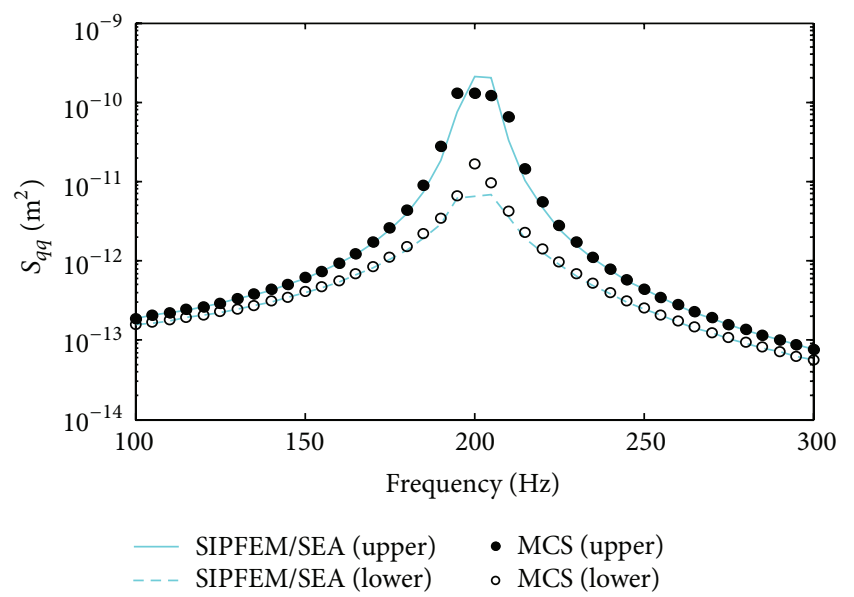

(c)

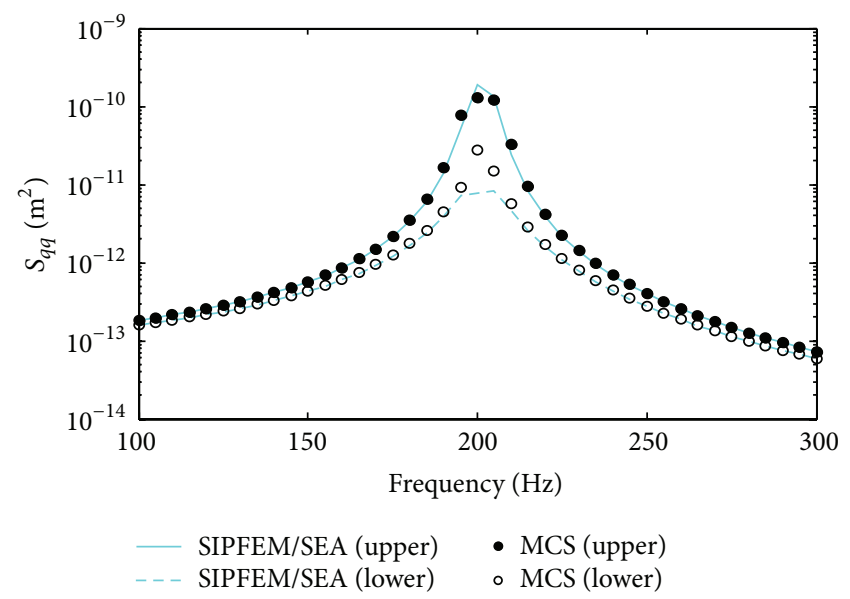

(b)

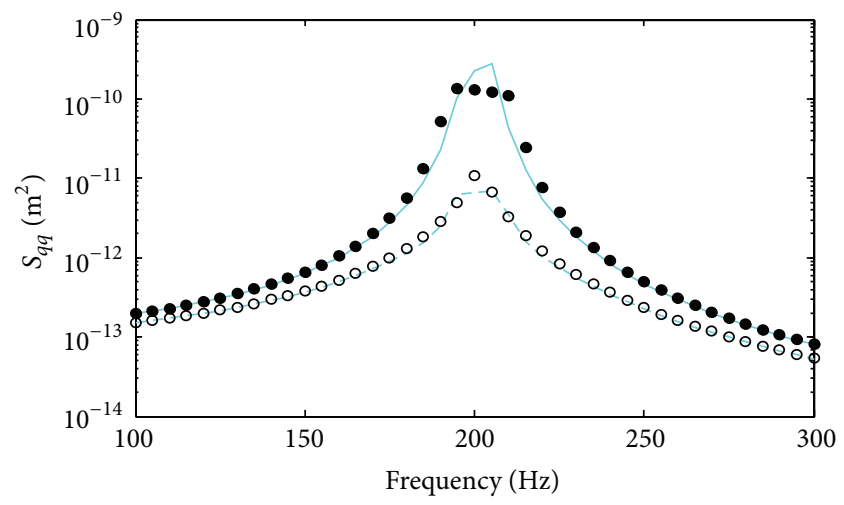

$\begin{array}{ll}\text { SIPFEM/SEA (upper) } & - \text { MCS (upper) } \\ --- \text { SIPFEM/SEA (lower) } & \circ \text { MCS (lower) }\end{array}$

(d)

FIgURE 3: The bounds of the autospectra of the mass displacements: (a) $\alpha=0.01$; (b) $\alpha=0.02$; (c) $\alpha=0.03$; (d) $\alpha=0.04$.

TABLE 6: The relative errors of the bounds of the plate energy and the autospectrum of the driving point displacements for different uncertainty levels $(f=330 \mathrm{~Hz})$.

\begin{tabular}{cccccccc}
\hline & \multirow{2}{*}{ Uncertainty level $\alpha$} & \multicolumn{3}{c}{ Lower bounds } & \multicolumn{3}{c}{ Upper bounds } \\
& & MCS & SIPFEM/SEA & Relative errors (\%) & MCS & SIPFEM/SEA & Relative errors (\%) \\
\hline \multirow{3}{*}{$E(\mathrm{~J})$} & 0.01 & $5.18 E-07$ & $5.11 E-07$ & 1.35 & $6.71 E-07$ & $6.68 E-07$ & 0.45 \\
& 0.02 & $4.70 E-07$ & $4.49 E-07$ & 4.47 & $7.89 E-07$ & $7.63 E-07$ & 3.30 \\
& 0.03 & $4.37 E-07$ & $3.99 E-07$ & 8.70 & $9.62 E-07$ & $8.70 E-07$ & 9.56 \\
& 0.04 & $4.11 E-07$ & $3.61 E-07$ & 12.17 & $1.20 E-06$ & $9.89 E-07$ & 17.58 \\
\hline \multirow{3}{*}{$S_{q q}\left(\mathrm{~m}^{2}\right)$} & 0.01 & $2.61 E-15$ & $2.61 E-15$ & 0 & $4.43 E-15$ & $4.43 E-15$ & 0 \\
& 0.02 & $2.10 E-15$ & $1.88 E-15$ & 10.48 & $5.84 E-15$ & $5.53 E-15$ & 5.31 \\
& 0.03 & $1.68 E-15$ & $1.27 E-15$ & 24.40 & $7.86 E-15$ & $6.75 E-15$ & 14.12 \\
& 0.04 & $1.33 E-15$ & $7.88 E-16$ & 40.75 & $1.08 E-14$ & $8.09 E-15$ \\
\hline
\end{tabular}

the bounds of the plate energy and the autospectra of the driving point displacements in the frequency band $f=200 \sim$ $600 \mathrm{~Hz}$ calculated by S-SIPFEM/SEA; the considered number of subintervals for each interval parameter is three. The corresponding relative errors of the bounds for frequencies $f=330 \mathrm{~Hz}$ and $f=440 \mathrm{~Hz}$ are shown in Table 8. From Figures $7(\mathrm{~d}), 8(\mathrm{~d}), 9$, and 10 and Tables 6 8, we can conclude that if the uncertainty level of the interval parameters increases to a certain value, SIPFEM/SEA will yield unreliable results. When SIPFEM/SEA yields unreliable results, S-SIPFEM/SEA can be used to improve the accuracy by gradually increasing the number of subintervals. If the number of subintervals is sufficiently large, S-SIPFEM/SEA will yield perfect results. 


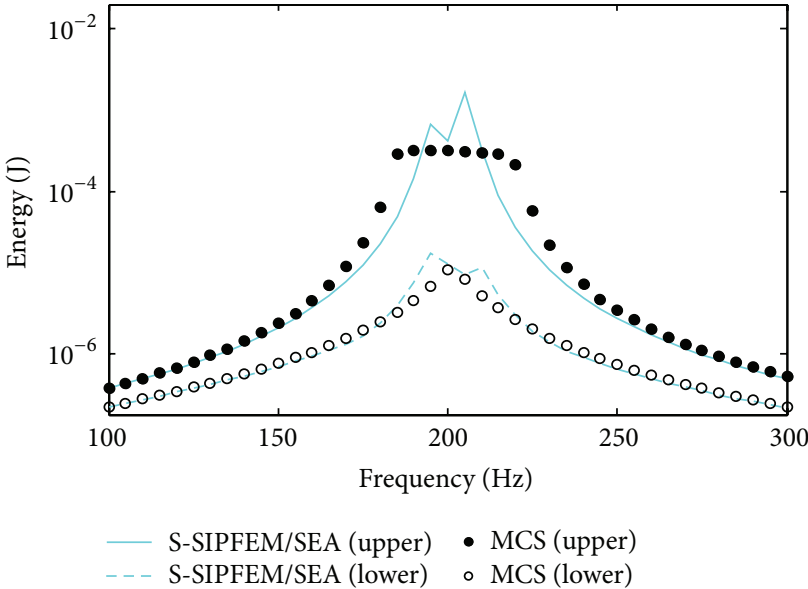

(a)

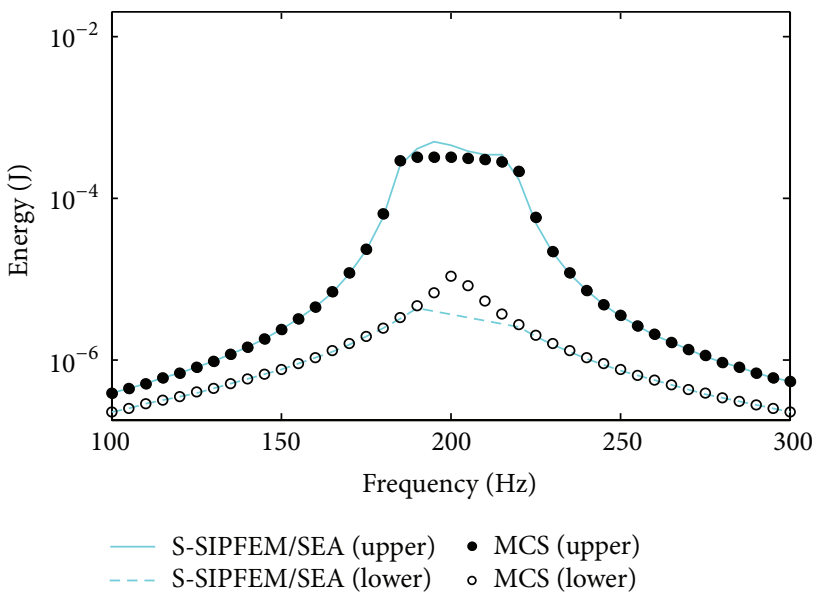

(c)

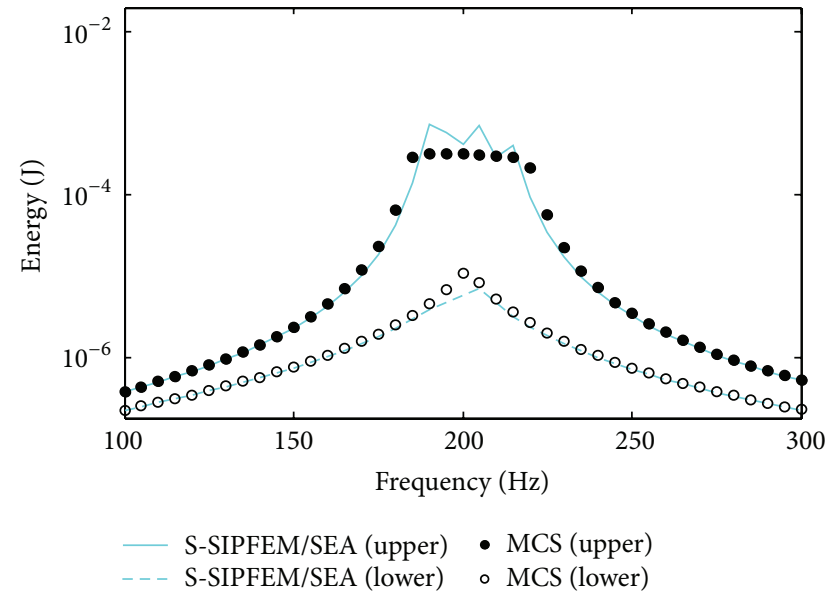

(b)

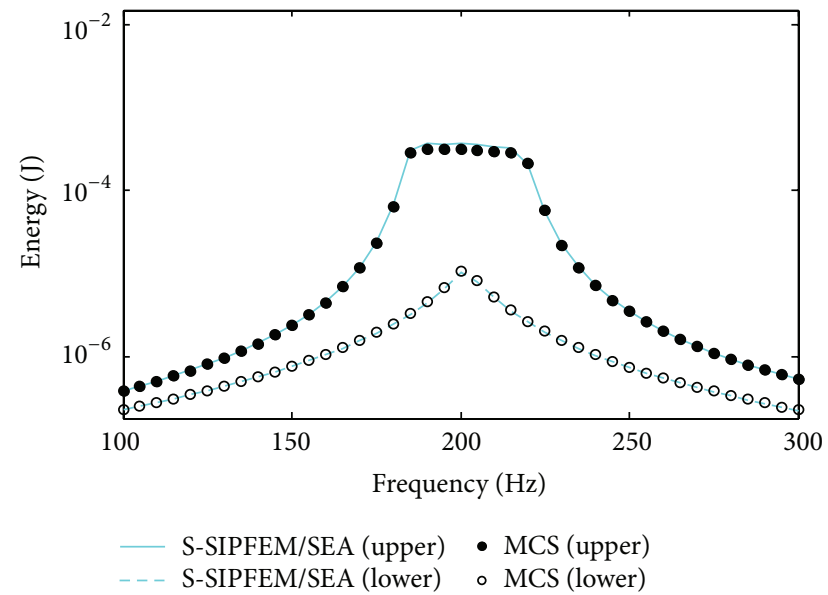

(d)

Figure 4: The bounds of the plate energy: (a) one subinterval, (b) two subintervals, (c) four subintervals, and (d) eight subintervals.

TABLE 7: The relative errors of the bounds of the plate energy and the autospectrum of the driving point displacements for different uncertainty levels $(f=440 \mathrm{~Hz})$.

\begin{tabular}{cccccccc}
\hline & \multirow{2}{*}{ Uncertainty level $\alpha$} & \multicolumn{3}{c}{ Lower bounds } & \multicolumn{2}{c}{ Upper bounds } \\
& & MCS & SIPFEM/SEA & Relative errors (\%) & MCS & SIPFEM/SEA & Relative errors (\%) \\
\hline \multirow{3}{*}{$E(\mathrm{~J})$} & 0.01 & $1.39 E-06$ & $1.38 E-06$ & 0.71 & $1.61 E-06$ & $1.61 E-06$ \\
& 0.02 & $1.30 E-06$ & $1.27 E-06$ & 2.31 & $1.74 E-06$ & $1.74 E-06$ & 0 \\
& 0.03 & $1.21 E-06$ & $1.17 E-06$ & 3.31 & $1.93 E-06$ & $1.87 E-06$ & 3.11 \\
& 0.04 & $1.14 E-06$ & $1.08 E-06$ & 5.26 & $2.11 E-06$ & $2.02 E-06$ & 4.27 \\
\hline \multirow{3}{*}{$S_{q q}\left(\mathrm{~m}^{2}\right)$} & 0.01 & $5.79 E-15$ & $5.79 E-15$ & 0 & $7.45 E-15$ & $7.45 E-15$ & 0 \\
& 0.02 & $5.28 E-15$ & $5.06 E-15$ & 4.17 & $8.44 E-15$ & $8.38 E-15$ & 0.71 \\
& 0.03 & $4.69 E-15$ & $4.40 E-15$ & 6.18 & $9.94 E-15$ & $9.38 E-15$ & 5.63 \\
& 0.04 & $4.28 E-15$ & $3.80 E-15$ & 11.21 & $1.14 E-14$ & $1.04 E-14$ \\
\hline
\end{tabular}

TABLE 8: The relative errors of the bounds of the plate energy and the autospectrum of the driving point displacements with three subintervals.

\begin{tabular}{cccccccc}
\hline & \multirow{2}{*}{ Frequency $(\mathrm{Hz})$} & \multicolumn{4}{c}{ Lower bounds } & \multicolumn{2}{c}{ Upper bounds } \\
& & MCS & S-SIPFEM/SEA & Relative errors (\%) & MCS & S-SIPFEM/SEA & Relative errors (\%) \\
\hline \multirow{2}{*}{$E(\mathrm{~J})$} & 330 & $4.11 E-07$ & $4.04 E-06$ & 1.70 & $1.20 E-06$ & $1.19 E-06$ & 0.83 \\
& 440 & $1.14 E-06$ & $1.12 E-06$ & 1.75 & $2.11 E-06$ & $2.12 E-05$ & 0.47 \\
\hline \multirow{2}{*}{$S_{q q}\left(\mathrm{~m}^{2}\right)$} & 330 & $1.33 E-15$ & $1.25 E-15$ & 6.02 & $1.08 E-14$ & $1.05 E-14$ & 2.78 \\
& 440 & $4.28 E-15$ & $4.15 E-13$ & 3.04 & $1.14 E-14$ & $1.15 E-12$ \\
\hline
\end{tabular}




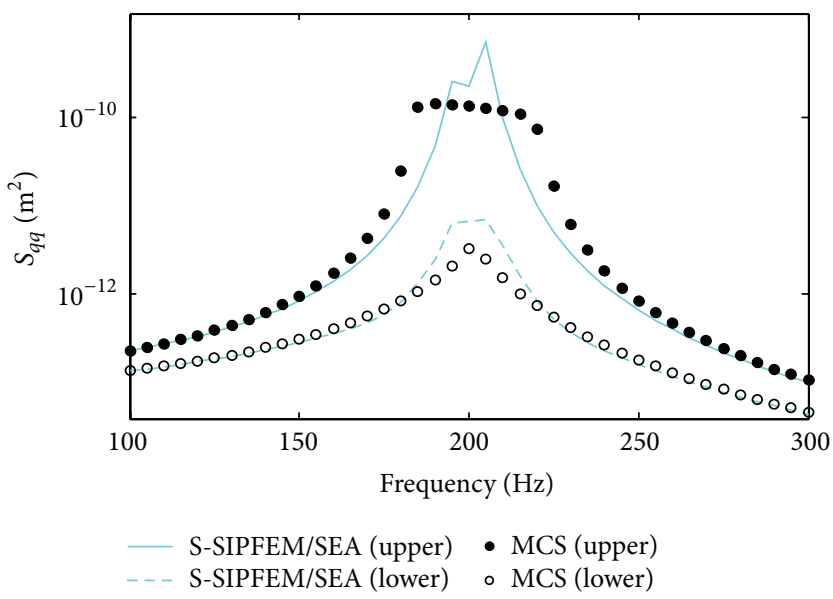

(a)

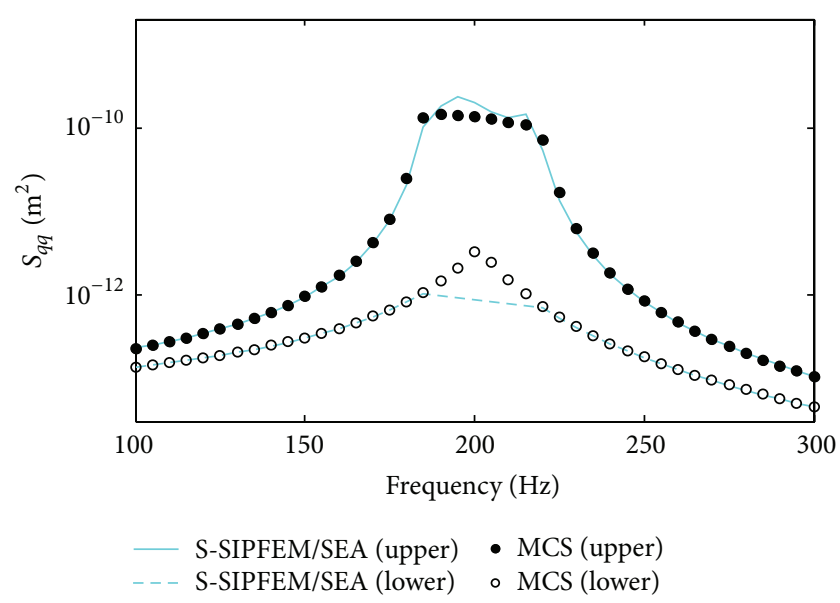

(c)

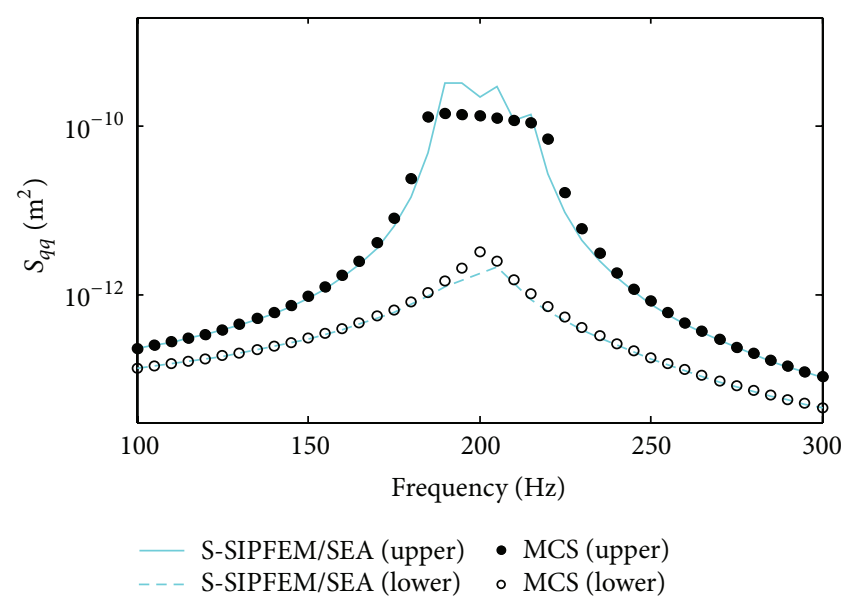

(b)

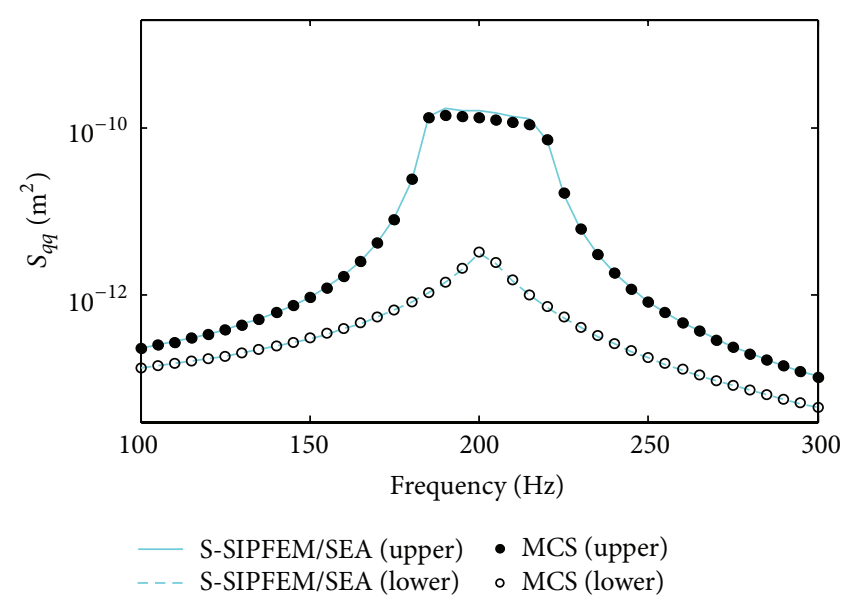

(d)

Figure 5: The bounds of the autospectra of the mass displacements: (a) one subinterval, (b) two subintervals, (c) four subintervals, and (d) eight subintervals.

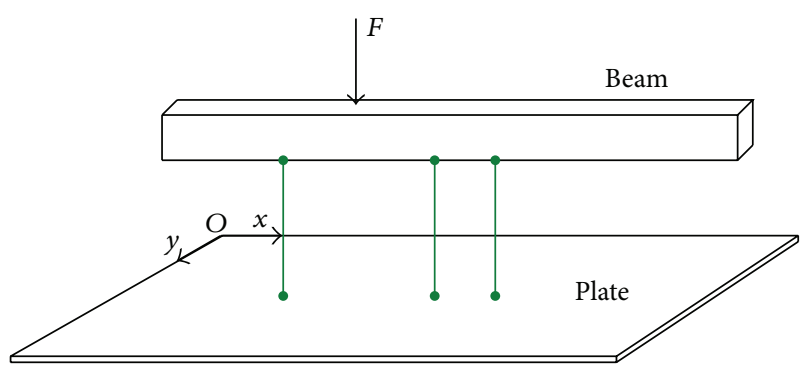

FIgURE 6: The beam-plate system.

Execution times of the S-SIPFEM/SEA with three subintervals and the Monte Carlo simulation of the hybrid FE/SEA model for calculating the plate energy at the same frequency are listed in Table 9. It can be seen from Table 9 that the SSIPFEM is much more time-saving than the Monte Carlo simulation of the hybrid FE/SEA model. It is foreseeable that the computational cost of S-SIPFEM/SEA will increase gradually with the increase of the number of subintervals, but
TABLE 9: Execution times of S-SIPFEM/SEA and the Monte Carlo simulation of the hybrid FE/SEA model.

\begin{tabular}{lcc}
\hline & MCS & S-SIPFEM/SEA \\
\hline Execution time(s) & 5,954 & 123 \\
\hline
\end{tabular}

in engineering practice, the number of subintervals will be restrained according to the required accuracy.

\section{Conclusions}

In order to improve the efficiency of midfrequency analysis of built-up structure systems with interval parameters, the second-order interval perturbation method and the subinterval technique are introduced into the FE/SEA framework in this study. Based on the FE/SEA equations for builtup structure systems and the second-order interval perturbation method, the frequency response variables (the SEA subsystem energy and the cross-spectrum of the response of the FE components, over the interval parametric and 


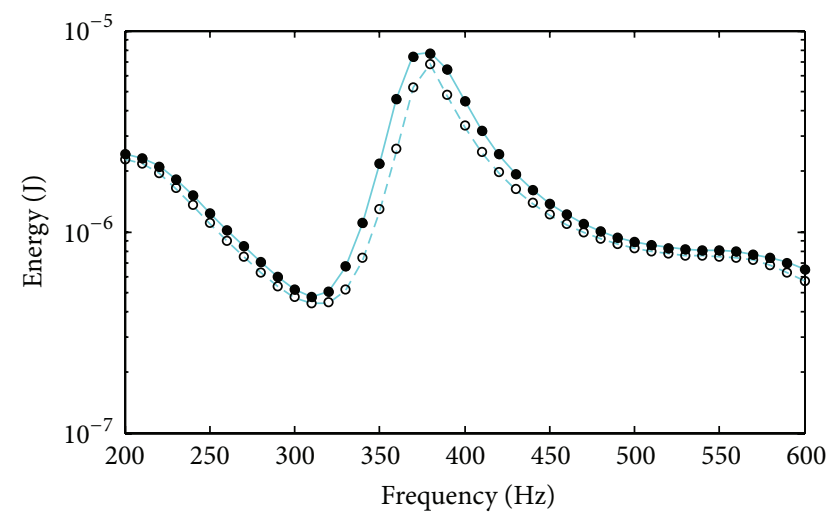

\begin{tabular}{ll} 
SIPFEM/SEA (upper) & MCS (upper) \\
\hline & SIPFEM/SEA (lower)
\end{tabular}

(a)

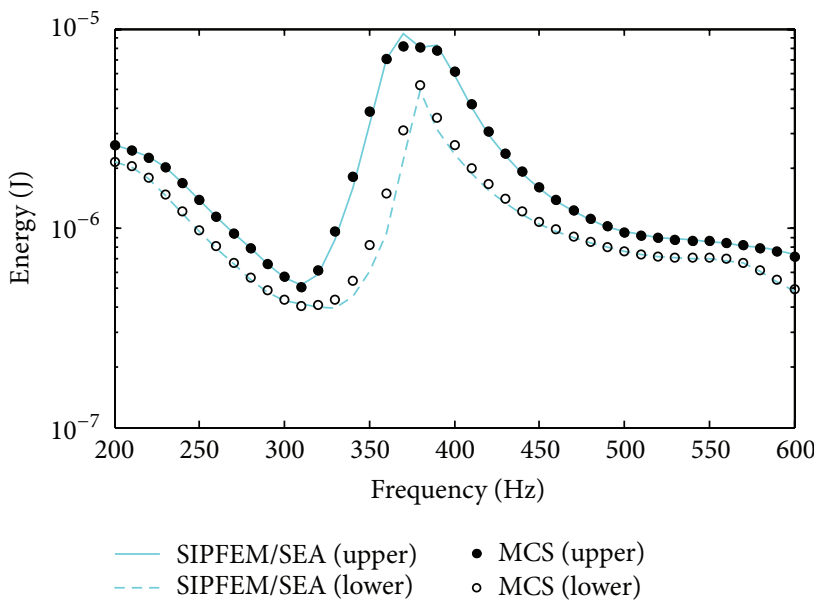

(c)

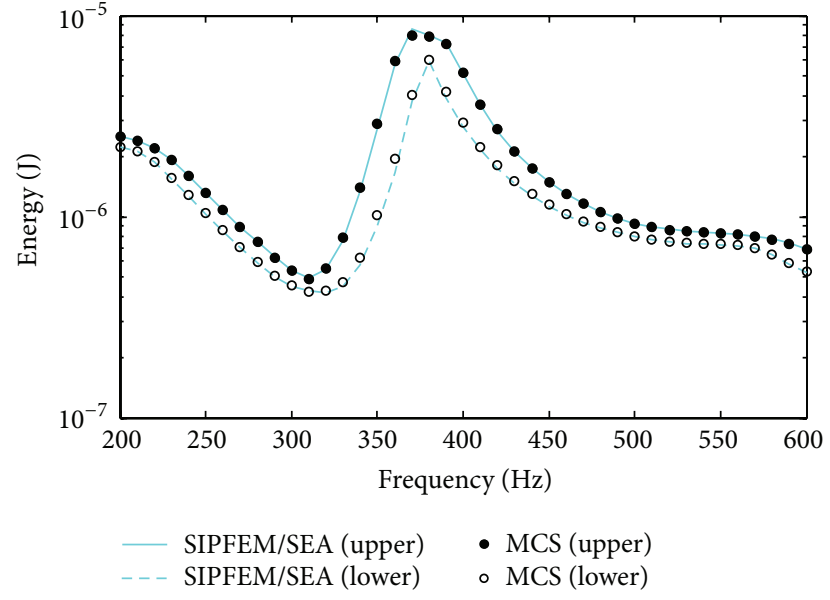

(b)

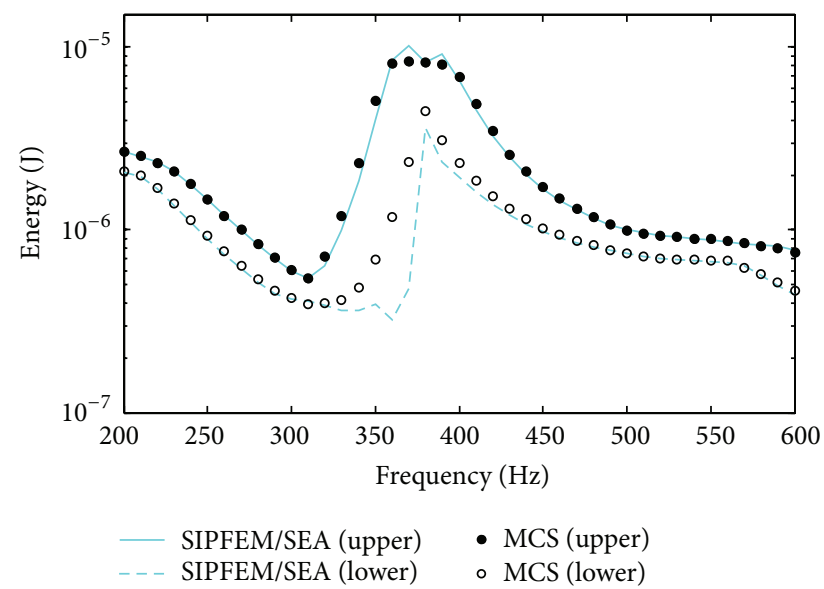

(d)

FIGURE 7: The bounds of the plate energy: (a) $\alpha=0.01$; (b) $\alpha=0.02$; (c) $\alpha=0.03$; (d) $\alpha=0.04$.

nonparametric uncertainties) are expanded with the secondorder Taylor series at the mean values of interval parameters. The nondiagonal elements of the Hessian matrices are neglected for the sake of simplicity and efficiency and thus the expanded response variables can be considered as the sum of a series of quadratic functions with respect to the interval parameters. By searching the target positions of the interval parameters that maximize or minimize the quadratic functions, the bounds of the ensemble averaged responses can be obtained. Due to the neglect of the higher order terms of Taylor series, SIPFEM/SEA is limited to the interval analysis of built-up systems with narrow parameter intervals. For the interval analysis of built-up systems with larger parameter intervals, the subinterval perturbation method based on the SIPFEM/SEA is introduced.

The proposed methods are illustrated by application to two built-up structure models, and reference results are obtained by the Monte Carlo simulations of the hybrid FE/SEA models. From the numerical results on the two builtup structure models, we conclude that (1) the accuracy of SIPFEM/SEA is remarkable for the midfrequency analysis of built-up structure systems with small interval parametric uncertainty; (2) the accuracy of SIPFEM/SEA decreases gradually with the increase of the interval parametric uncertainty, and if the uncertainty level increases to a certain value, unreliable results will be yielded. This is mainly because of the unpredictable and uncontrollable effect arising from the neglect of the higher order terms of Taylor series; (3) the subinterval perturbation method based on SIPFEM/SEA can be efficiently applied to the midfrequency analysis of built-up structure systems with large interval parametric uncertainty, and the accuracy of S-SIPFEM/SEA can be improved monotonically by increasing the number of subintervals. Thus, we can determine that SIPFEM/SEA and the subinterval perturbation method based on SIPFEM/SEA are powerful techniques for the midfrequency analysis of builtup structure systems with interval parametric uncertainty of the FE components.

\section{Conflict of Interests}

The authors declare that there is no conflict of interests regarding the publication of this paper. 


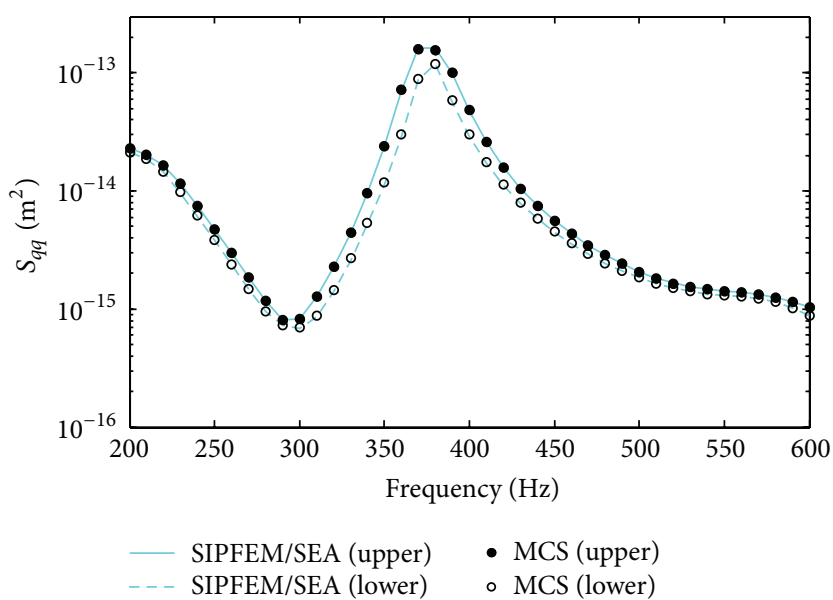

(a)

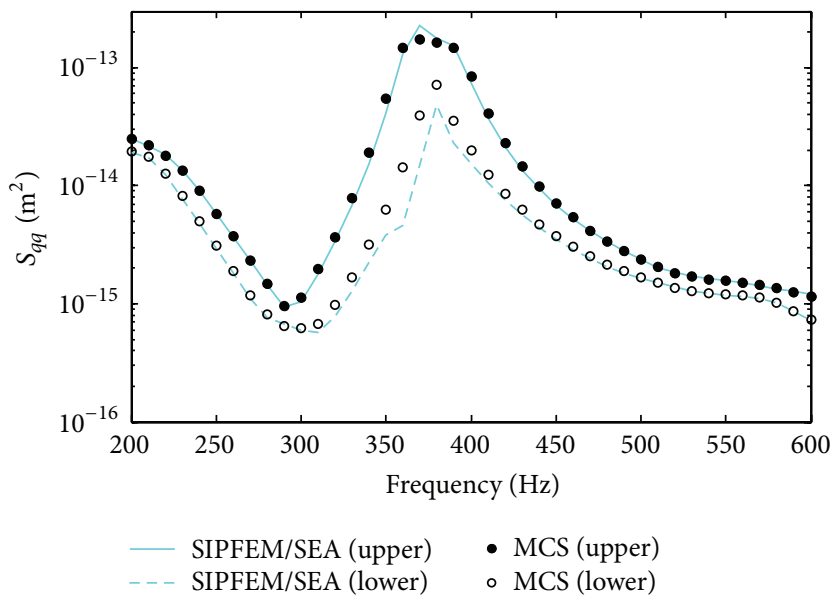

(c)

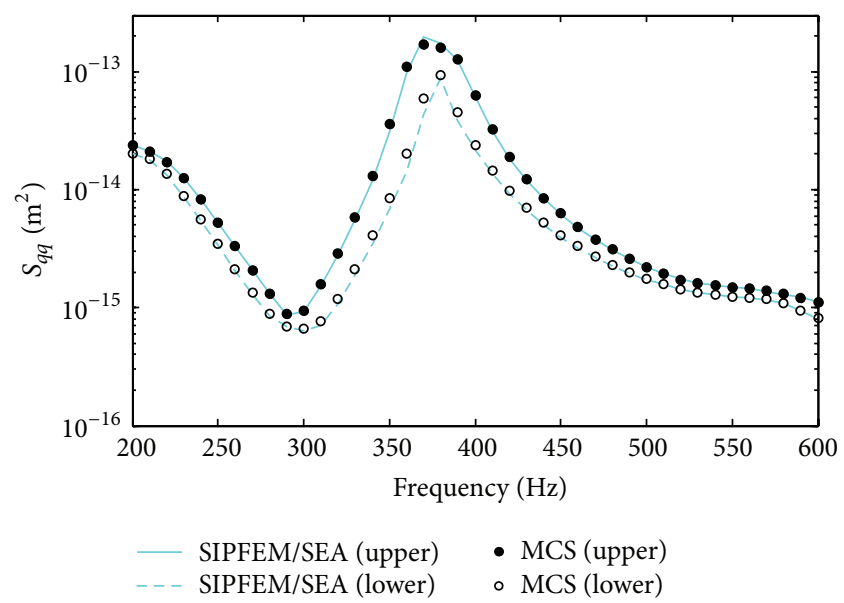

(b)

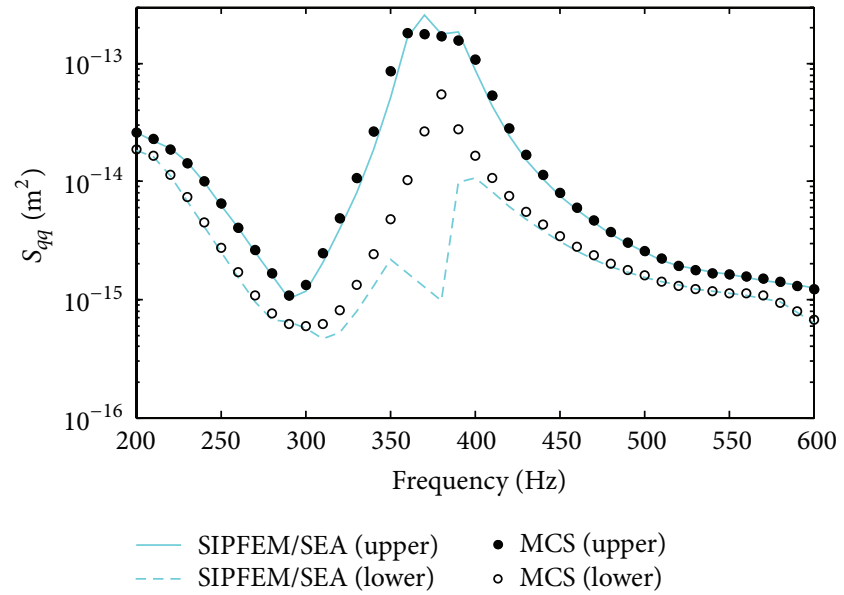

(d)

FIGURE 8: The bounds of the autospectra of the driving point displacements: (a) $\alpha=0.01$; (b) $\alpha=0.02$; (c) $\alpha=0.03$; (d) $\alpha=0.04$.

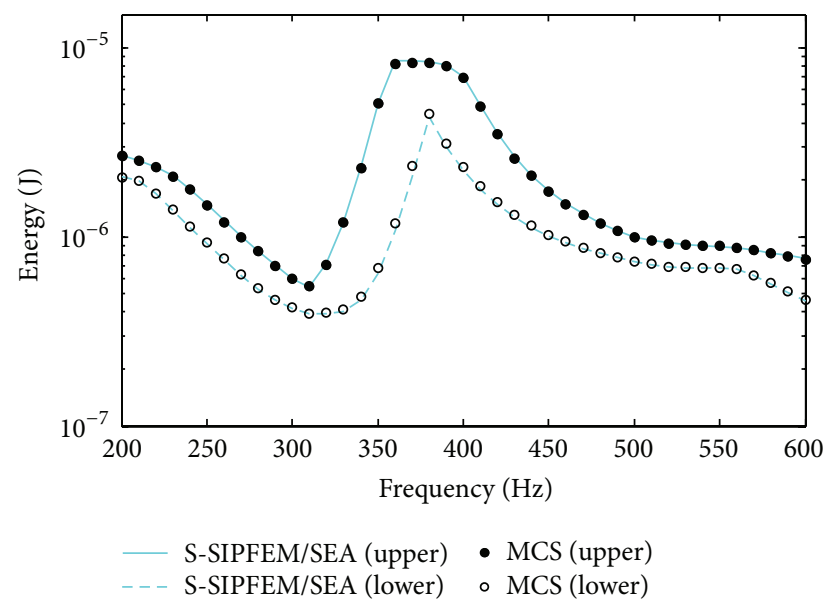

FIgURE 9: The bounds of the plate energy with three subintervals.

\section{Acknowledgments}

The paper is supported by National Natural Science Foundation of China (no. 11402083) and Independent Research Projects of State Key Laboratory of Advanced Design and

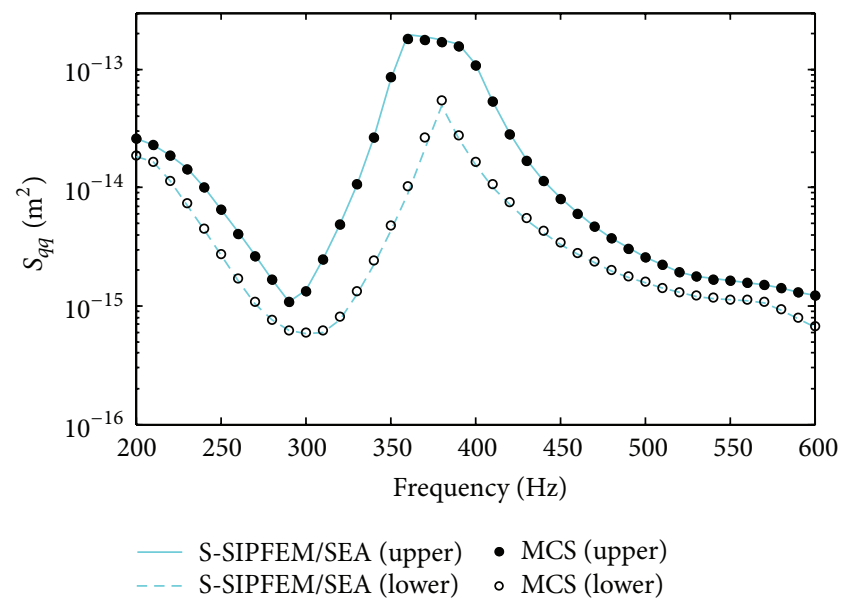

FIgURE 10: The bounds of the autospectra of the driving point displacements with three subintervals.

Manufacturing for Vehicle Body in Hunan University (Grants no. 734215002 and no. 51375002). 


\section{References}

[1] D. Moens and D. Vandepitte, "Recent advances in nonprobabilistic approaches for non-deterministic dynamic finite element analysis," Archives of Computational Methods in Engineering. State of the Art Reviews, vol. 13, no. 3, pp. 389-464, 2006.

[2] T. D. Hien and M. Kleiber, "Finite element analysis based on stochastic Hamilton variational principle," Computers and Structures, vol. 37, no. 6, pp. 893-902, 1990.

[3] B. Z. Xia and D. J. Yu, "Interval analysis of acoustic field with uncertain-but-bounded parameters," Computers and Structures, vol. 112-113, pp. 235-244, 2012.

[4] Y. T. Feng, C. F. Li, and D. R. J. Owen, "A directed Monte Carlo solution of linear stochastic algebraic system of equations," Finite Elements in Analysis and Design, vol. 46, no. 6, pp. 462473, 2010.

[5] G. Stefanou, "The stochastic finite element method: past, present and future," Computer Methods in Applied Mechanics and Engineering, vol. 198, no. 9-12, pp. 1031-1051, 2009.

[6] O. C. Zienkiewicz, R. L. Taylor, J. Z. Zhu, and P. Nithiarasu, The Finite Element Method-The Three Volume Set, ButterworthHeinemann, 6th edition, 2005.

[7] K. Vergote, B. Van Genechten, D. Vandepitte, and W. Desmet, "On the analysis of vibro-acoustic systems in the mid-frequency range using a hybrid deterministic-statistical approach," Computers and Structures, vol. 89, no. 11-12, pp. 868-877, 2011.

[8] R. H. Lyon and R. G. DeJong, Theory and Application of Statistical Energy Analysis, Butterworth-Heinemann, 2nd edition, 1995.

[9] P. Ladevèze, L. Arnaud, P. Rouch, and C. Blanze, “The variational theory of complex rays for the calculation of mediumfrequency vibrations," Engineering Computations, vol. 18, no. 12, pp. 193-214, 2001.

[10] P. Ladevèze, L. Blanc, P. Rouch, and C. Blanzé, "A multiscale computational method for medium-frequency vibrations of assemblies of heterogeneous plates," Computers and Structures, vol. 81, no. 12, pp. 1267-1276, 2003.

[11] W. Desmet, A wave based prediction technique for coupled vibroacoustic analysis [Ph.D. thesis], Katholieke Universiteit, Division PMA, Leuven, Belgium, 1998.

[12] B. Pluymers, B. Van Hal, D. Vandepitte, and W. Desmet, "Trefftz-based methods for time-harmonic acoustics," Archives of Computational Methods in Engineering. State of the Art Reviews, vol. 14, no. 4, pp. 343-381, 2007.

[13] C. Soize, "A model and numerical method in the medium frequency range for vibroacoustic predictions using the theory of structural fuzzy," The Journal of the Acoustical Society of America, vol. 94, no. 2, pp. 849-865, 1993.

[14] P. J. Shorter and R. S. Langley, "Vibro-acoustic analysis of complex systems," Journal of Sound and Vibration, vol. 288, no. 3, pp. 669-699, 2005.

[15] P. J. Shorter and R. S. Langley, "On the reciprocity relationship between direct field radiation and diffuse reverberant loading," Journal of the Acoustical Society of America, vol. 117, no. 1, pp. 85-95, 2005.

[16] V. Cotoni, P. J. Shorter, and R. S. Langley, "Numerical and experimental validation of a hybrid finite element-statistical energy analysis method," Journal of the Acoustical Society of America, vol. 122, no. 1, pp. 259-270, 2007.

[17] E. Reynders and R. S. Langley, "Response probability distribution of built-up vibro-acoustic systems," Journal of the Acoustical Society of America, vol. 131, no. 2, pp. 1138-1149, 2012.
[18] R. S. Langley and V. Cotoni, "Response variance prediction for uncertain vibro-acoustic systems using a hybrid deterministicstatistical method," Journal of the Acoustical Society of America, vol. 122, no. 6, pp. 3445-3463, 2007.

[19] R. S. Langley and P. J. Shorter, "The wave transmission coefficients and coupling loss factors of point connected structures," Journal of the Acoustical Society of America, vol. 113, no. 4 I, pp. 1947-1964, 2003.

[20] A. Cicirello and R. S. Langley, "The vibro-acoustic analysis of built-up systems using a hybrid method with parametric and non-parametric uncertainties," Journal of Sound and Vibration, vol. 332, no. 9, pp. 2165-2178, 2013.

[21] C. Soize, "Stochastic modeling of uncertainties in computational structural dynamics-recent theoretical advances," Journal of Sound and Vibration, vol. 332, no. 10, pp. 2379-2395, 2013.

[22] B. MacE, W. Desmet, and B. Pluymers, "Mid-frequency methods in sound and vibration-part A," Journal of Sound and Vibration, vol. 332, no. 8, pp. 1895-1896, 2013.

[23] B. Mace, W. Desmet, and B. Pluymers, "Mid-frequency methods in sound and vibration-part B," Journal of Sound and Vibration, vol. 332, no. 9, p. 2131, 2013.

[24] A. Cicirello and R. S. Langley, "Efficient parametric uncertainty analysis within the hybrid Finite Element/Statistical Energy Analysis method," Journal of Sound and Vibration, vol. 333, no. 6, pp. 1698-1717, 2014.

[25] I. Doltsinis and Z. Kang, "Perturbation-based stochastic FE analysis and robust design of inelastic deformation processes," Computer Methods in Applied Mechanics and Engineering, vol. 195, no. 19-22, pp. 2231-2251, 2006.

[26] B. Xia, D. Yu, and J. Liu, “Transformed perturbation stochastic finite element method for static response analysis of stochastic structures," Finite Elements in Analysis and Design, vol. 79, pp. 9-21, 2014.

[27] W. Gao, N. Zhang, and J. Ji, "A new method for random vibration analysis of stochastic truss structures," Finite Elements in Analysis and Design, vol. 45, no. 3, pp. 190-199, 2009.

[28] S. Shang and G. J. Yun, "Stochastic finite element with material uncertainties: implementation in a general purpose simulation program," Finite Elements in Analysis and Design, vol. 64, pp. 65-78, 2013.

[29] A. Neumaier, Interval Methods for Systems of Equations, Cambridge, UK, Cambridge University Press, Cambridge, 1990.

[30] Z. P. Qiu, Y. Y. Xia, and J. L. Yang, “The static displacement and the stress analysis of structures with bounded uncertainties using the vertex solution theorem," Computer Methods in Applied Mechanics and Engineering, vol. 196, no. 49-52, pp. 4965-4984, 2007.

[31] Z. P. Qiu, S. H. Chen, and I. Elishakoff, "Bounds of eigenvalues for structures with an interval description of uncertain-butnon-random parameters," Chaos, Solitons \& Fractals, vol. 7, no. 3, pp. 425-434, 1996.

[32] Z. Qiu and I. Elishakoff, "Antioptimization of structures with large uncertain-but-non-random parameters via interval analysis," Computer Methods in Applied Mechanics and Engineering, vol. 152, no. 3-4, pp. 361-372, 1998.

[33] S. H. Chen, L. Ma, G. W. Meng, and R. Guo, "An efficient method for evaluating the natural frequencies of structures with uncertain-but-bounded parameters," Computers and Structures, vol. 87, no. 9-10, pp. 582-590, 2009.

[34] K. Fujita and I. Takewaki, "An efficient methodology for robustness evaluation by advanced interval analysis using updated 
second-order Taylor series expansion," Engineering Structures, vol. 33, no. 12, pp. 3299-3310, 2011.

[35] B. Xia, D. Yu, and J. Liu, "Interval and subinterval perturbation methods for a structural-acoustic system with interval parameters," Journal of Fluids and Structures, vol. 38, pp. 146-163, 2013.

[36] Z. Qiu, L. Ma, and X. Wang, "Unified form for static displacement, dynamic response and natural frequency analysis based on convex models," Applied Mathematical Modelling, vol. 33, no. 10, pp. 3836-3847, 2009.

[37] N. Impollonia and G. Muscolino, "Interval analysis of structures with uncertain-but-bounded axial stiffness," Computer Methods in Applied Mechanics and Engineering, vol. 200, no. 21-22, pp. 1945-1962, 2011.

[38] R. S. Langley and V. Cotoni, "The ensemble statistics of the vibrational energy density of a random system subjected to single point harmonic excitation," Journal of the Acoustical Society of America, vol. 118, no. 5, pp. 3064-3076, 2005.

[39] L. Ji and Y. H. Zhen, "Analysis of subsystem randomness effects on the mid-frequency vibrations of built-up structures," Journal of Sound and Vibration, vol. 332, no. 13, pp. 3190-3200, 2013. 

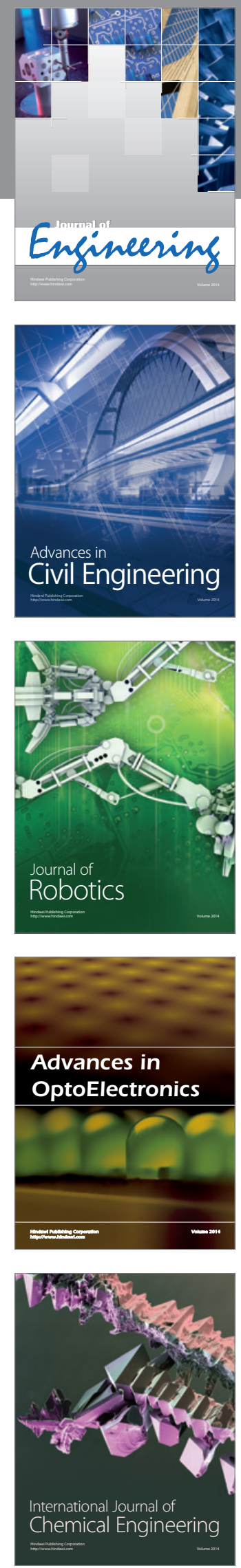

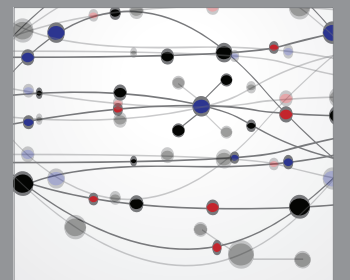

The Scientific World Journal
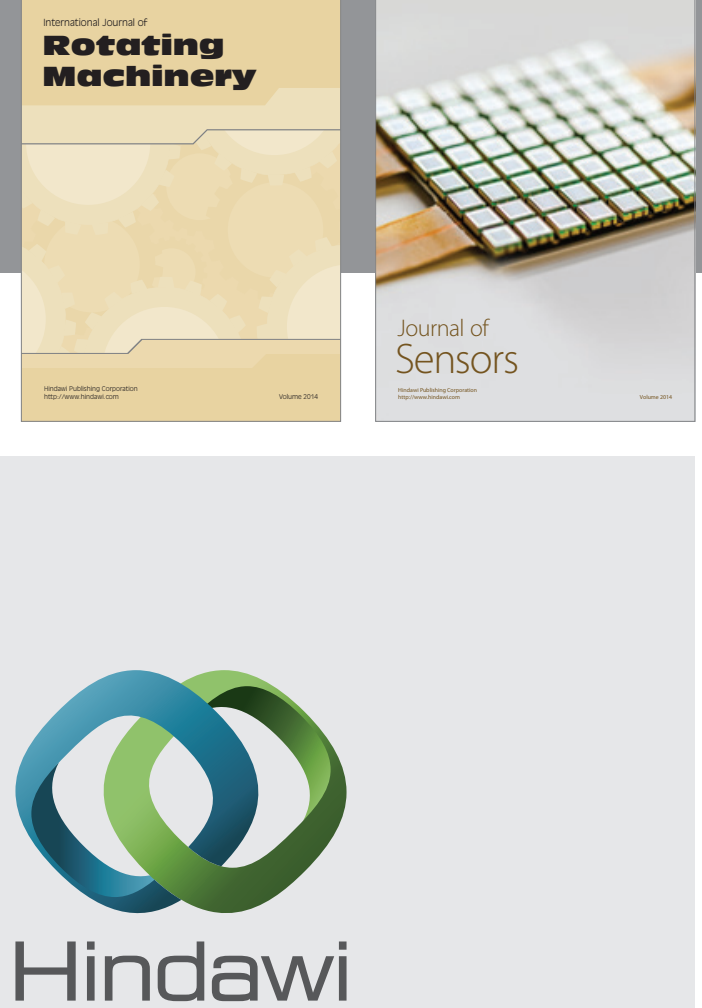

Submit your manuscripts at http://www.hindawi.com
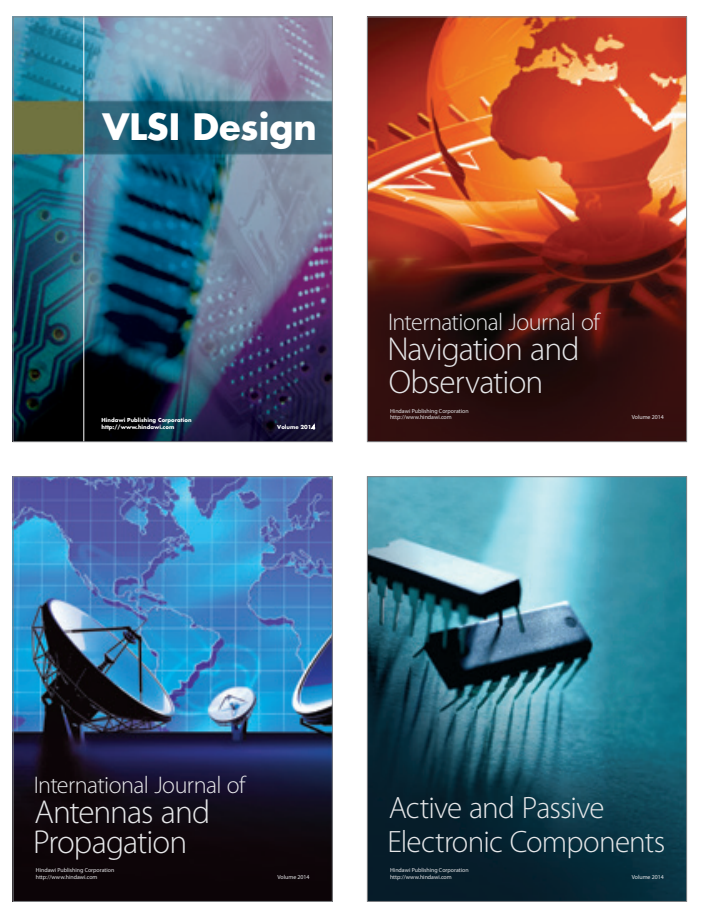
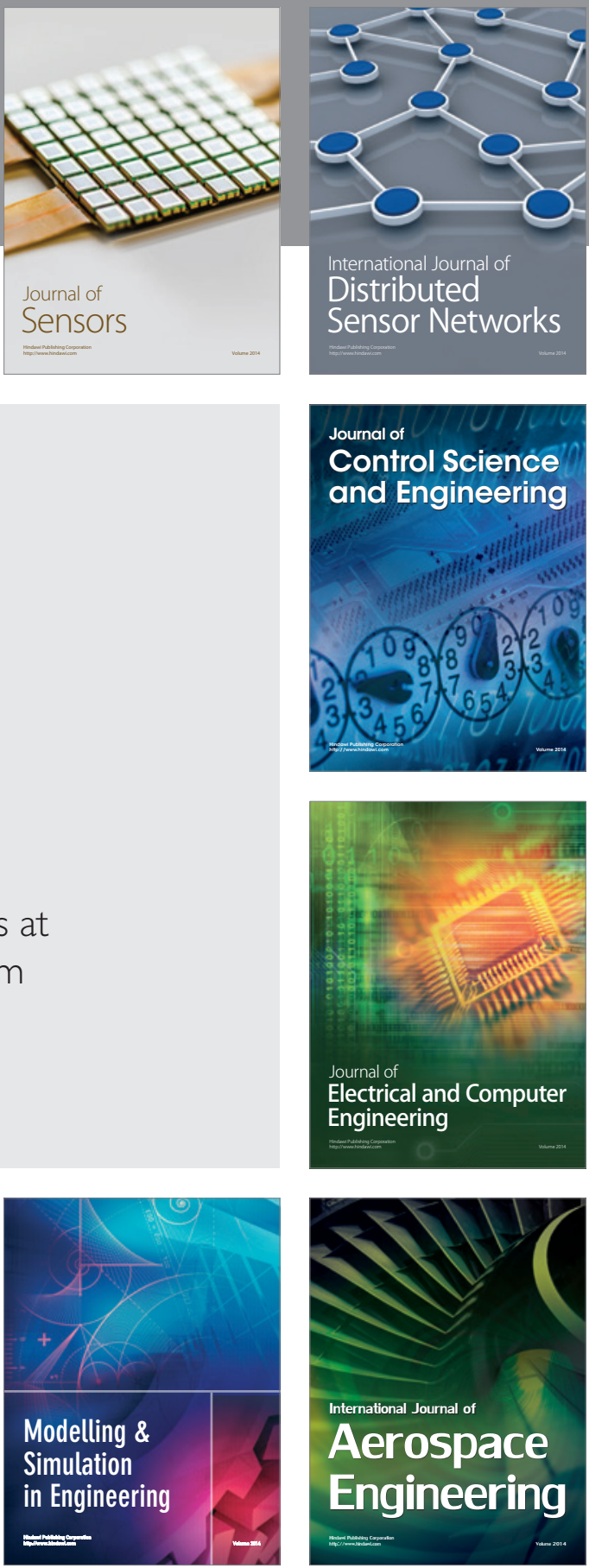

Journal of

Control Science

and Engineering
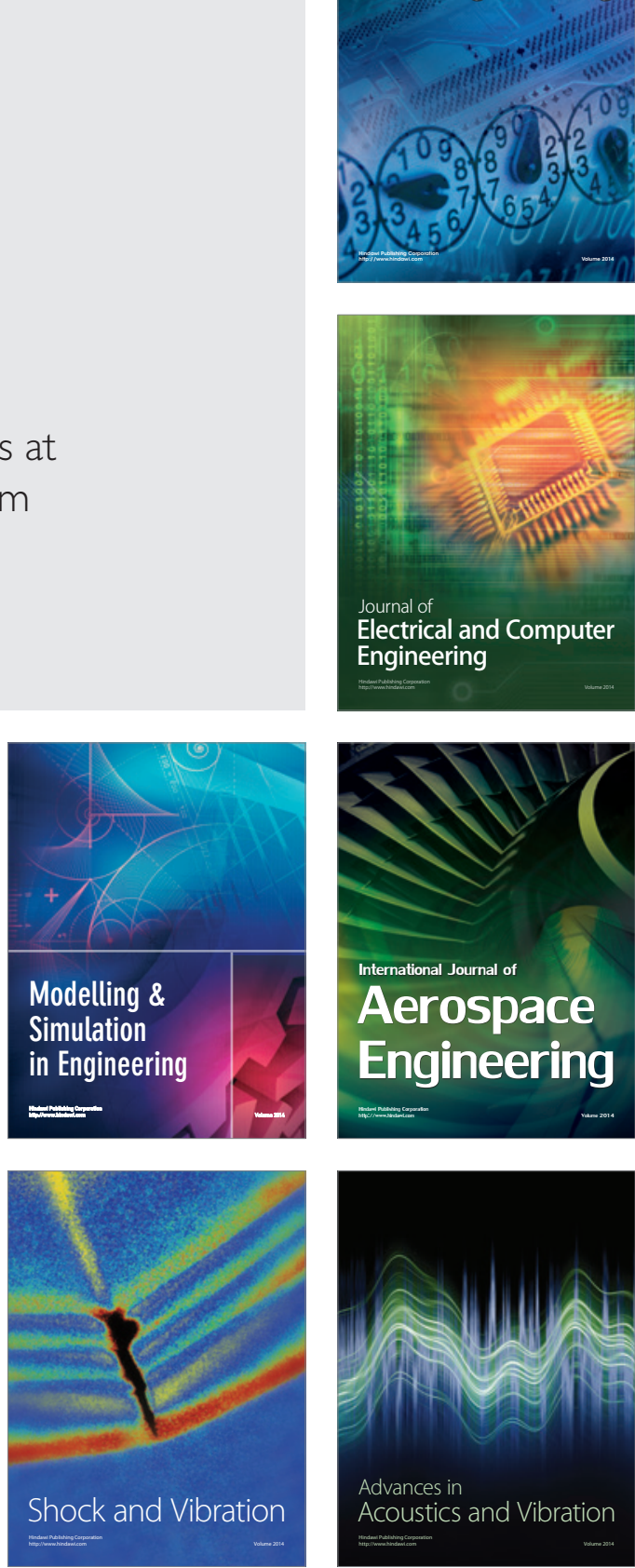